\title{
基于三苯胺的苂光探针设计、合成与应用研究进展
}

\author{
陈思鸿 ${ }^{a}$ 陈 淇 ${ }^{a}$ 罗时荷 $*, a, b$ 曹西颖 ${ }^{a}$ \\ 杨国贤 $a$ 曾晓晴 $a$ 汪朝阳*,a,b \\ ( ${ }^{a}$ 华南师范大学化学学院 教育部环境理论化学重点实验室 广州市生物医药分析化学重点实验室 广州 510006) \\ ( $b$ 华南理工大学广东省功能分子工程重点实验室 广州 510640)
}

\begin{abstract}
摘要 螺旋桨结构的三苯胺荧光团既能作为强的电子供体, 又能作为潜在的聚集诱导发光(AIE)骨架. 同时, 三苯胺衍 生物很容易通过简单的反应进行结构修饰, 如醛基、氨基、硼酸基、卤素、乙炔基等取代的三苯胺能够发生缩合反应 或偶联反应等, 进一步功能化. 因此, 功能性三苯胺类化合物被广泛用于太阳能电池、荧光染料、固态发光材料和荧光 探针的分子设计中. 根据三苯胺基荧光探针的检测对象, 将其分为阳离子、阴离子和中性小分子荧光探针三类, 并从分 子的结构和性能出发, 重点综述了近五年来国内外三苯胺基苂光探针在分子设计、合成与检测应用方面的最新进展. 展望未来，构建近红外发光和高量子效率的 AIE 苂光探针值得关注.
\end{abstract}

关键词 三苯胺荧光团; 苂光探针; 设计策略; 合成路线; 传感机理; 检测应用

\section{Progress in Design, Synthesis and Application of Triphenylamine-Based Fluorescent Probes}

\author{
Chen, Sihong $^{a} \quad$ Chen, $^{a}{ }^{a} \quad$ Luo, Shihe ${ }^{*, a, b} \quad$ Cao, Xiying ${ }^{a}$ \\ Yang, Guoxian $^{a} \quad$ Zeng, Xiaoqing $^{a} \quad$ Wang, Zhaoyang ${ }^{*, a, b}$
}

$\left({ }^{a}\right.$ Key Laboratory of Theoretical Chemistry of Environment, Ministry of Education, Guangzhou Key Laboratory of Analytical Chemistry for Biomedicine, School of Chemistry, South China Normal University, Guangzhou 510006)

$\left({ }^{b}\right.$ Key Lab of Functional Molecular Engineering of Guangdong Province in SCUT, Guangzhou 510640)

\begin{abstract}
The triphenylamine fluorophore with propeller-structure can be used not only as a strong electron donor, but also as a potential aggregation-induced emission (AIE) framework. At the same time, triphenylamine derivatives with different substituted groups (e.g. formyl, amino, halogen, boronic acid group, alkynyl) can easily be modified for further functionalization by simple reactions, such as condensation and metal coupling reaction. Thus, the functional triphenylamine derivatives are widely applied in the molecular design of solar cells, fluorescent dyes, solid-state luminescent materials and fluorescent probes. According to the detected analytes, such as cations, anions, and neutral small molecules, the triphenylamine-based fluorescent probes reported in the past five years are divided into three types. And on the viewpoint of their structures and performances, the new progress on the molecular design, synthesis and detecting application is reviewed. The developing potential of triphenylamine-based fluorescent probes is also envisioned, and the AIE-type probes with the properties of near-infrared and high photoluminescence quantum yield should be highlighted in the future.
\end{abstract}

Keywords triphenylamine fluorophore; fluorescent probe; design strategy; synthetic route; sensing mechanism; detection application

作为常见的电子供体, 螺旋桨结构的三苯胺单元被 广泛应用于构建太阳能电池材料 ${ }^{[1-2]}$ 、聚集诱导发光材 料 ${ }^{[3-4]}$ 和苂光探针分子 ${ }^{[5-6]}$. 其中, 基于三苯胺的小分子
苂光探针不仅具有斯托克斯位移大、苂光量子产率高以 及光稳定性良好等优点，而且能够高选择性地识别痕量 的金属阳离子 ${ }^{[7]} 、$ 阴离子 ${ }^{[8]} 、$ 生物硫醇 ${ }^{[9]}$ 和芳香爆炸物 ${ }^{[10]}$

* Corresponding authors. E-mail: wangzy@scnu.edu.cn; pinky_r@163.com

Received September 5, 2020; revised September 29, 2020; published online October 21, 2020.

Project supported by the Scientific Special Project of Guangzhou Science and Technology (No. 201607010251), the Science and Technology Project of Guangdong Province (No. 2017A010103016) and the Open Fund of the Key Laboratory of Functional Molecular Engineering of Guangdong Province (No. $2017 \mathrm{kf01}$.

广州市科技计划科学研究专项(No. 201607010251)、广东省科技计划(No. 2017A010103016)及广东省功能分子工程重点实验室(2017kf01)资助项目. 
等多种多样的分析物, 迅速成为近年的研究热点. 但是, 鲜有关于三苯胺类苂光探针的文献综述报道. 鉴于此, 本文依据三苯胺类苂光探针检测对象的不同，从分子设 计、光学性能与检测机理的角度, 对近五年的三苯胺基 苂光探针的研究进展进行了综述.

\section{1 基于三苯胺的阳离子荧光探针}

许多金属离子在我们的日常生活中起着至关重要 的作用, 如铝离子、铜离子、铁离子和锌离子等, 它们 的存在与生物体的生理健康息息相关. 然而, 一旦过量, 也是有害的. 同时，一些重金属离子是有毒的，如录离 子和银离子等, 会导致严重的健康和环境问题. 因此, 设计合成特异性识别痕量金属阳离子的苂光探针受到 了广泛的关注 ${ }^{[11-12]}$.

\section{1 应用于单个阳离子的检测}

第一个金属阳离子苂光探针的问世可追溯到 1867 年, GoppelsrÖder 等 ${ }^{[13]}$ 报道了用于检测铝离子的螯合荧 光增强型探针. 至今为止, 大多数已报道的金属阳离子 的苂光探针设计原理是基于主客体之间的协同相互作 用 ${ }^{[14]}$. 其中, 用于检测金属离子的三苯胺探针也不例 外 $^{[15]}$.

铝是地壳中第三丰富的元素, 常见于食品添加剂、 铝炊具和抗胃酸药物中. 世界卫生组织(WHO)建议每人
每天摄入 $\mathrm{Al}^{3+}$ 大约 3 10 mg, 超过限定的食用范围会 导致肌萎缩性侧索硬化、帕金森病和阿尔茨海默病等不 可逆疾病 ${ }^{[16]}$. 因此, 用于检测 $\mathrm{Al}^{3+}$ 的三苯胺类苂光探针 常有报道 ${ }^{[17]}$. 周虹屏课题组 ${ }^{[18]}$ 构建了两个基于三苯胺 的双支链席夫碱苂光探针 1 和 2 , 其结构上分别通过碳 碳双键、碳碳单键桥连(Scheme 1), 故分别与 $\mathrm{Al}^{3+}$ 结合 时有两种不同的作用机理. 在 $\mathrm{CH}_{3} \mathrm{OH} / \mathrm{H}_{2} \mathrm{O}(V: V=4$ :

1)混合溶液中，探针 $\mathbf{1}$ 和 $\mathrm{Al}^{3+}$ 相互作用，先发生金属促 进席夫碱水解再与 $\mathrm{N}$ 原子螯合, 形成明亮蓝绿色苂光的 $1+\mathrm{Al}^{3+}$ 配合物. 探针 2 苯酚上的 $\mathrm{O}$ 原子与 $\mathrm{Al}^{3+}$ 配位后, 直接通过螯合增强苂光作用(CHEF)形成亮绿色苂光的 $2+\mathrm{Al}^{3+}$ 配合物. 这可能是因为探针 2 中的单键可自由 旋转，两个羟基的空间距离非常适合与 $\mathrm{Al}^{3+}$ 相互作用， 但探针 1 的双键刚性结构使两个羟基向外，难以与两个 羟基直接发生配位作用. 有趣的是，虽然探针 $\mathbf{1}$ 和 $\mathbf{2}$ 的 作用机理不同，但是具有差不多的检测限(LOD)，分别 是 $1.22 \times 10^{-7}$ 和 $2.99 \times 10^{-7} \mathrm{~mol} / \mathrm{L}$. 在应用上，两个探针 不仅能在水介质中选择性识别 $\mathrm{Al}^{3+}$, 还均可通过 “turn on” 型苂光信号，以非侵入性的方式穿透细胞膜，有效 地检测 HepG2 细胞中的外源性 $\mathrm{Al}^{3+}$.

铜是人体中第三位不可缺少的微量元素，是生物体 内必不可少的过渡金属, 因为它参与各种生理和病理过 程. 许多疾病与铜含量的失衡有关，如 Menkes 综合征

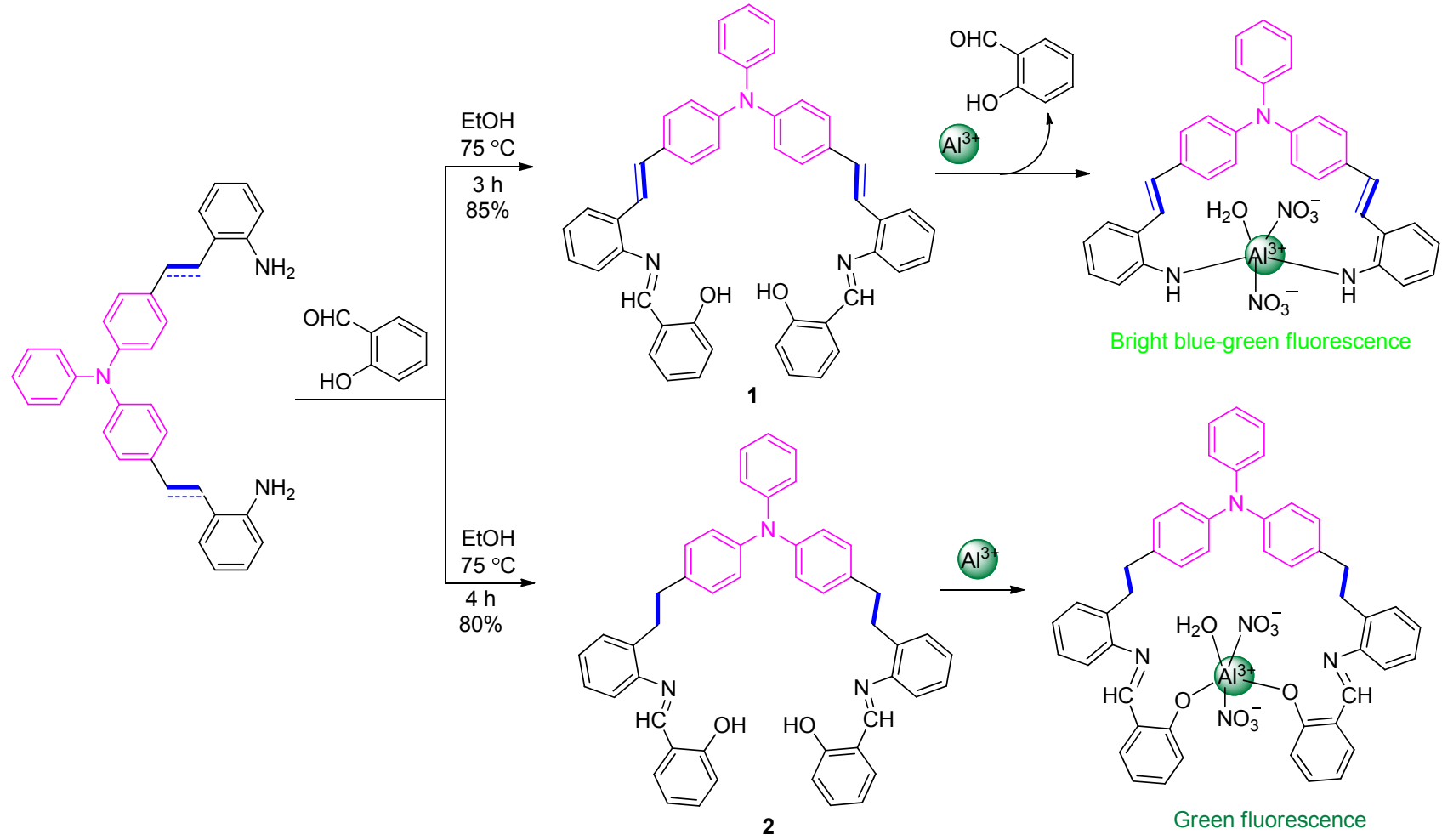

图式 1 探针 1 和 2 的合成与作用机理

Scheme 1 Synthesis and sensing mechanism of probes $\mathbf{1}$ and $\mathbf{2}$ 
(铜缺乏)、威尔逊病(铜过量)、朊病毒病及神经退行性 变等 ${ }^{[19]}$. 美国环境保护署(EPA)已经规定了饮用水中铜 离子的最大浓度为 $20 \mu \mathrm{mol} / \mathrm{L}$, 故发展快速检测环境样 品中铜离子的探针引起了广泛关注 ${ }^{[20]}$. 蒲守智课题 组 ${ }^{[21]}$ 设计并合成了一种以六氟环戊烯为受体, 吡嗪和 三苯胺为供体, 2-(亚氨基甲基)苯酚为 $\pi$ 桥的新型荧光探 针 3 (Scheme 2). 在探针 3 的四氢呋喃(THF)溶液中, 加 入 $\mathrm{Cu}^{2+}$ 后, 苯酚上的 $\mathrm{O}$ 原子以及席夫碱和吡嗪的 $\mathrm{N}$ 原 子与 $\mathrm{Cu}^{2+}$ 发生 $1: 1$ 络合, 激发态分子内质子转移 (ESIPT)过程受到抑制, 螯合增强苂光猝灭(CHEQ)效应 被激活, 3 的橙色苂光变成黑色. 但是, 加入其它金属离 子时, 溶液苂光无明显变化. 不仅如此, 探针 $\mathbf{3}$ 还可在 实际水样中高精度检测 $\mathrm{Cu}^{2+}$.

最近, 沈建良课题组 ${ }^{[22]}$ 以 4-醛基三苯胺(A)和 2-水 杨酰肼为原料合成了线型荧光探针 4 (Scheme 3). 在苂 光探针 4 的 $\mathrm{DMSO} / \mathrm{H}_{2} \mathrm{O}(V: V=1: 1)$ 的混合溶液中加 入 $\mathrm{Cu}^{2+}$ 后, 席夫碱上的 $\mathrm{N}$ 原子和羰基上的 $\mathrm{O}$ 原子与 $\mathrm{Cu}^{2+}$ 形成一个 $2: 1$ 的复合物, 发生金属-配体电荷转移 (MLCT), 导致明显的紫外吸收光谱红移; 同时, 分子内 电荷转移(ICT)受到抑制, 蓝色苂光被明显猝灭. 不仅如 此, 探针 4 具有较高的特异性和生物相容性, 还能在 HepG2、A549 和 HeLa 细胞中成功实现 $\mathrm{Cu}^{2+}$ 可视化检
测和成像.

铁是地壳中含量丰富的元素之一, $\mathrm{Fe}^{3+}$ 是人体和植 物必需的离子. 但是, $\mathrm{Fe}^{3+}$ 的过量和缺乏都会导致各种 机能障碍和疾病, 如贫血和失眠等神经退行性疾病 ${ }^{[23]}$. 因此, 开发高选择性的 $\mathrm{Fe}^{3+}$ 苂光探针具有十分重要的意 $义^{[24]}$. 通过缩合反应，醛基取代的三苯胺和 2-氨基苯酚 常被用来合成席夫碱荧光探针, 用于检测金属离子. 霍 延平课题组 ${ }^{[25]}$ 设计合成的蓝色菼光探针 5 (图 1)成功通 过光诱导电子转移机制(PET)机制实现了活细胞中 $\mathrm{Fe}^{3+}$ 的检测.

若以三苯胺单元作为电子供体, 三氮唑结构为电子 受体，也可通过 Suzuki 偶联反应构筑蓝色荧光探针. 张 明课题组 ${ }^{[26]}$ 构建了三个电子供体-电子受体(D-A)型蓝 光分子 $6 \sim 8$ (图 1). 该 D-A 型化合物具有分子内电荷转 移(ICT)机制，同时三氮唑裸露的 $\mathrm{N}$ 原子对 $\mathrm{Fe}^{3+}$ 具有较 高的络合能力, 与 $\mathrm{Fe}^{3+}$ 形成复合物影响分子间电荷转 移, 导致蓝色荧光猝灭, 故可将 $6 \sim 8$ 开发为 $\mathrm{Fe}^{3+}$ 苂光探 针, 其中探针 7 具有最低的 $\operatorname{LOD}\left(1.2 \times 10^{-7} \mathrm{~mol} / \mathrm{L}\right)$.

锌是人体第二丰富的微量元素, 主要分布在人体细 胞和组织液中, 游离锌离子的失调与神经系统疾病和细 胞调亡密切相关 ${ }^{[27]}$. 因此, 有效监测和快速检测生物样

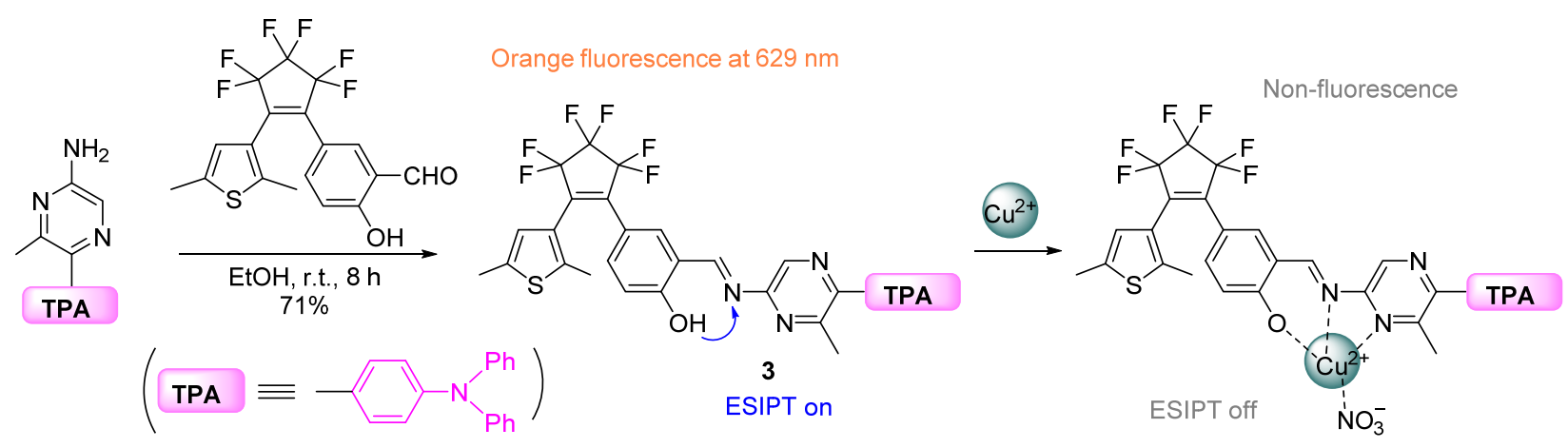

图式 2 探针 3 的合成与作用机理

Scheme 2 Synthesis and sensing mechanism of probe 3<smiles>CC(C)[R16](=O)OCc1ccccc1C(=O)NN</smiles>

A

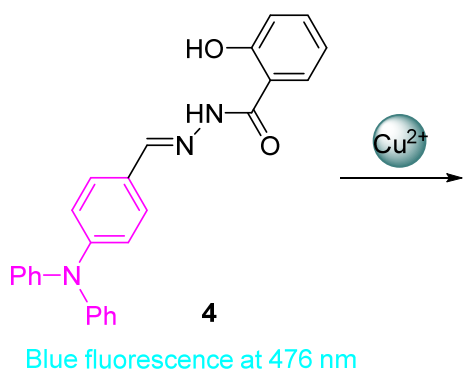

图式 3 探针 4 的合成与作用机理

Scheme 3 Synthesis and sensing mechanism of probe 4 


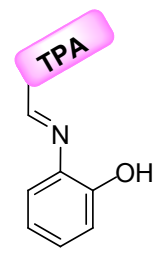

5

At $470 \mathrm{~nm}$

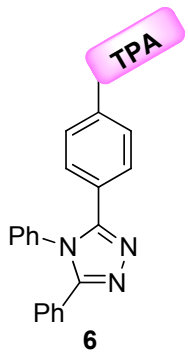

At $460 \mathrm{~nm}$

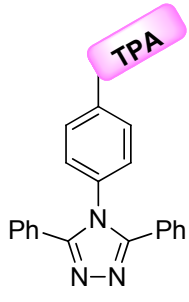

7

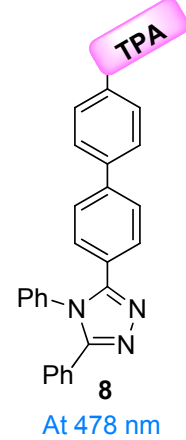

图 1 蓝色荧光探针 $5 \sim 8$ 的分子结构

Figure 1 Molecular structures of blue fluorescent probes $5 \sim 8$

品中的 $\mathrm{Zn}^{2+}$ 具有重要意义 ${ }^{[28]}$. 王宏青课题组 ${ }^{[29]}$ 合成了 新型三苯胺席夫碱苂光化合物 9 (Scheme 4), 利用酰肼 骨架金属螯合能力强、水溶性好、毒性低和生物活性高 的特点, 以及三苯胺结构的强紫外吸收、任意发光特性, 将化合物 9 开发成裸眼识别 $\mathrm{Zn}^{2+}$ 的苂光探针, 不仅适用 于活细胞成像，且能制备成测试纸条用于现场检测.
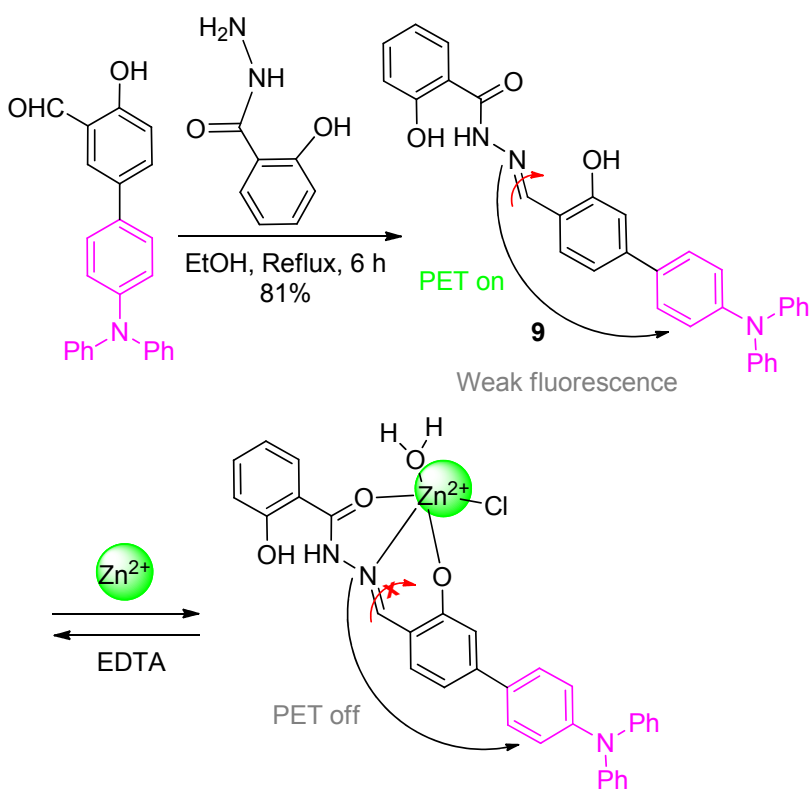

Yellow-green fluorescence at $540 \mathrm{~nm}$

图式 4 探针 9 的合成与作用机理

Scheme 4 Synthesis and sensing mechanism of probe 9

采是地球上最常见的致命毒素之一, 它有许多来 源, 如黄金生产、燃煤电厂、温度计、气压计和录灯 ${ }^{[30]}$. 在过去的几十年里, 已经开发了大量的用于检测 $\mathrm{Hg}^{2+}$ 的苂光探针 ${ }^{[31]}$. 常见的三苯胺型 $\mathrm{Hg}^{2+}$ 荧光探针对 $\mathrm{Hg}^{2+}$ 的特异性识别是利用 $\mathrm{Hg}^{2+}$ 的亲硫特性实现的 ${ }^{[32]}$. 例如, 通过脱硫反应使二乙基硫代缩醛恢复芳香醛结构，从而 产生明显的苂光响应 ${ }^{[33]}$. 为了更有效改善探针分子的 水溶性以及构建 ICT 分子延长发射波长, 肖海波课题
组 ${ }^{[34]}$ 通过在 9,9'-螺比芴的联苯分支上引入强供电子三 苯胺和强吸电子的二羧甲基硫代缩醛基团，制备了一种 新型 $\mathrm{Hg}^{2+}$ 苂光探针 10. 在其 $\mathrm{DMSO} / \mathrm{H}_{2} \mathrm{O}(V: V=1: 1)$ 的混合溶液中, 加入不同的金属离子, 只有 $\mathrm{Hg}^{2+}$ 与探针 作用而释放出醛基(Scheme 5), 苂光发射峰减弱并蓝移, 蓝色苂光被明显猝灭. 不仅如此, 在日光灯下，探针溶 液颜色随着 $\mathrm{Hg}^{2+}$ 加入由淡黄色变成无色, 能实现肉眼 检测. 此外, 探针 $\mathbf{1 0}$ 作为 ICT 分子, 能对 $\mathrm{Hg}^{2+}$ 实现双光 子苂光细胞成像，具有很好的应用前景.

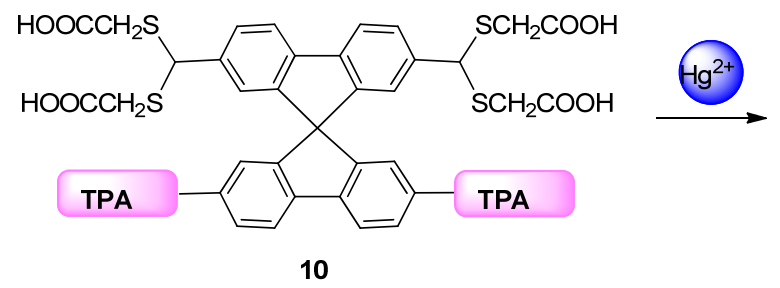

Blue fluorescence at $470 \mathrm{~nm}$

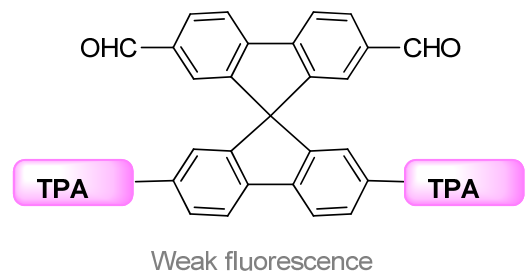

图式 5 探针 10 与 $\mathrm{Hg}^{2+}$ 的作用机理

Scheme 5 Sensing mechanism of probe $\mathbf{1 0}$

银在电子、照相、成像等行业中具有广阔的应用前 景, 银离子对环境, 特别是对生物的负面影响已引起人 们的广泛关注. 银离子可与各种代谢物和酶结合, 导致 酶的失活和随之而来的健康问题 ${ }^{[35]}$. 因此，找到一种简 单、快速的方法来检测和分析 $\mathrm{Ag}^{+}$具有非常重要的意 $义^{[36]}$. 考虑到三苯胺结构具有良好的苂光性和易修饰 性，可在适当的位置与外部识别基团相连，开发一系列 具有一定功能的苂光探针. 例如, 张献课题组 ${ }^{[37]}$ 以双醛 基取代的三苯胺和 1,10-菲罗啉-5,6-二酮为原料, 合成 了具有 $\mathrm{A}-\pi-\mathrm{D}-\pi-\mathrm{A}$ 结构的 $\mathrm{Ag}^{+}$苂光探针 11 (Eq. 1). 在探 针 11 的 Tris- $\mathrm{HCl}$ 缓冲溶液中, 逐渐加入 $\mathrm{Ag}^{+}$后, $440 \mathrm{~nm}$ 处的苂光发射峰增强, 这可能是探针菲罗啉骨架上的 $\mathrm{N}$ 原子与 $\mathrm{Ag}^{+}$形成 1: 1 的配合物所致. 重要的是, 探针 11 能在活细胞中对 $\mathrm{Ag}^{+}$实现双光子成像.

铬被广泛应用于油漆、炼钢、焊接、制革及催化剂 等领域，其广泛使用导致了铬污染严重，对生态系统和 生物安全产生了明显的不利影响 ${ }^{[38]}$. 铬主要存在两种 氧化态. 其中, $\mathrm{Cr}(\mathrm{III})$ 是人体必需的微量元素; 而 $\mathrm{Cr}(\mathrm{VI})$ 进入机体后, 由于难以去除和代谢缓慢, 细胞会发生癌 变. WHO 将饮用水中的 $\mathrm{Cr}(\mathrm{VI})$ 浓度限制为 $50 \mu \mathrm{g} / \mathrm{L}$, 故 


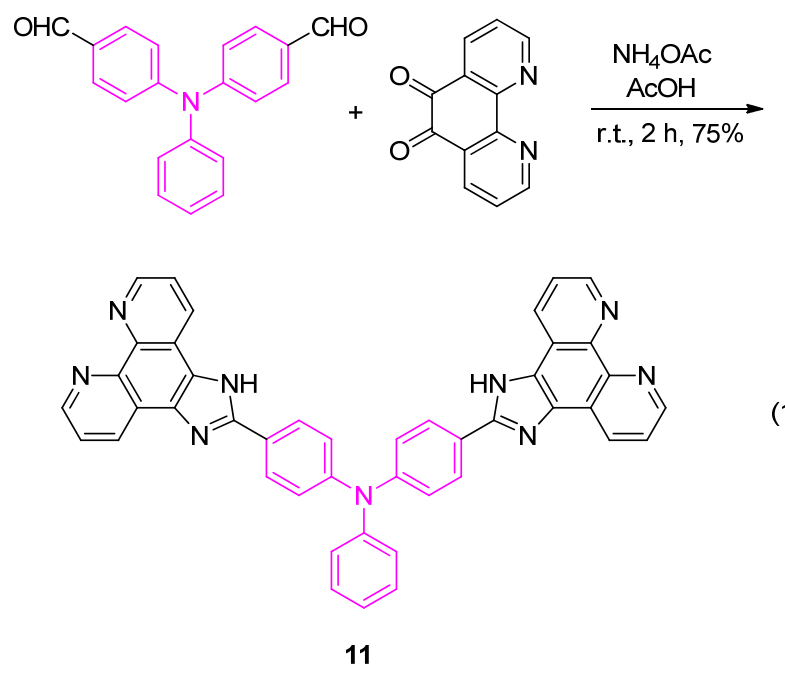

(1)

开发简单高效的 $\operatorname{Cr}(\mathrm{VI})$ 苂光探针具有重要意义. 林伟英 课题组 ${ }^{[39]}$ 设计并合成了一种新的脂滴靶向 $\mathrm{Cr}(\mathrm{VI})$ 苂光 探针 12 (Scheme 6), 其由亲油性的三苯胺醛基衍生物 $\mathbf{A}$ 和具有良好生物相容性的有机硅氧烷反应生成. 在探针 12 的磷酸盐缓冲液(PBS)溶剂中, 逐渐加入 $\mathrm{Cr}(\mathrm{VI}), 365$ $\mathrm{nm}$ 处的吸收峰值逐渐增大, 溶液颜色由无色变为黄色; 同时, $450 \mathrm{~nm}$ 处的苂光强度逐渐降低, 蓝色荧光明显猝 灭. 探针 12 的响应机制是在 $\mathrm{C}=\mathrm{N}$ 基团上的 $\mathrm{N}$ 原子与 $\mathrm{Cr}(\mathrm{VI})$ 发生配位相互作用, 破坏能量传递过程, 导致探 针苂光猝灭. 12 表现出优异的光稳定性, 且能够对 HeLa 细胞中的 $\mathrm{Cr}(\mathrm{VI})$ 具有较高的选择性和灵敏度.

\section{2 应用于多个阳离子的检测}

多功能(或双功能)荧光探针能通过相同或不同的作 用位点与多个分析物作用, 作用后不同程度地影响探针 分子的电荷分布, 从而发生明显的紫外和苂光光谱变 化 ${ }^{[40]}$. 多检测的苂光探针能提高检测效率、降低分析成 本, 极大地增强了化学探针的应用潜力 ${ }^{[41-42]}$. 在基于三 苯胺骨架的荧光探针中, 多个阳离子的检测也受到广泛 的关注 ${ }^{[43-44]}$. 其中, 基于三苯胺和席夫碱结构的双功能 荧光探针最为常见, 这是因为席夫碱结构既可充当分析 物的水解反应位点 ${ }^{[45-46]}$, 又可充当结合位点与阳离子发 生配位作用 ${ }^{[47-48]}$.

潘忠稳课题组 ${ }^{[49]}$ 利用星形三苯胺作为苂光团和水 杨醛分子作为识别受体, 构建了树枝状苂光探针 13 (Scheme 7), 其能在单一溶剂中同时灵敏、选择性地检 测 $\mathrm{Al}^{3+}$ 和 $\mathrm{Cu}^{2+}$. 探针 13 在乙醇溶液中, 发出绿色的苂 光, 加入 $\mathrm{Al}^{3+}$ 后诱导亚胺键水解, 荧光发射峰蓝移且增 强; 而 $\mathrm{Cu}^{2+}$ 与亚胺和羟基氧配位, 荧光发射峰蓝移且减 弱. 因此, 通过不同的作用机理, 探针 13 实现了两种离 子的特异性识别. 此外, 探针 13 还成功可视化检测 Hela 细胞、斑马鱼和拟南芥组织中累积的 $\mathrm{Al}^{3+}$ 和 $\mathrm{Cu}^{2+}$,

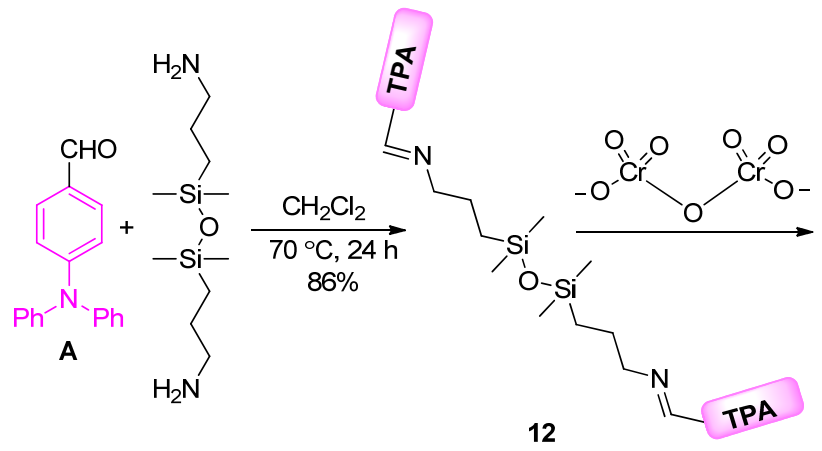

Blue fluorescence $450 \mathrm{~nm}$

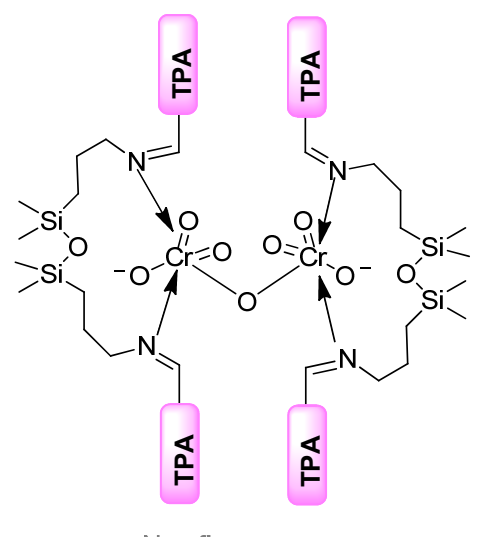

Non-fluorescence

图式 6 探针 12 的合成与作用机理

Scheme 6 Synthesis and sensing mechanism of probe $\mathbf{1 2}$

有望成为研究活体组织中离子的生理和病理作用的强 有力的分子工具.

类似地, 钱鹰课题组 ${ }^{[50]}$ 通过 Click 反应合成了基于 三苯胺、氟喼二吡咯(BODIPY)荧光染料和席夫碱的近 红外(NIR)苂光探针 14(图 2), 并通过水解反应和配位作 用两种不同的识别模式选择性检测 $\mathrm{Hg}^{2+}$ 和 $\mathrm{Fe}^{3+}$. 在 $\mathrm{THF} / \mathrm{H}_{2} \mathrm{O}(V: V=1: 1)$ 的缓冲溶液中, 苂光探针 14 在 510、598 和 $670 \mathrm{~nm}$ 处显示出三个发射峰, 但由于 $\mathrm{C}=\mathrm{N}$ 异构化和旋转引起的非辐射衰变, 其荧光强度相对较 弱. 当加入 $\mathrm{Fe}^{3+}$ 或 $\mathrm{Hg}^{2+}$ 时, $\mathrm{Hg}^{2+}$ 和 $\mathrm{Fe}^{3+}$ 都能促进席夫碱 的水解，使 $\mathrm{C}=\mathrm{N}$ 基团转化为甲酰基，导致探针的荧光 明显增强, 溶液颜色从紫色变为明显的绿色. 另外, $\mathrm{Fe}^{3+}$ 还与 BODIPY 核 3,5 位连接的 4-羟基苯乙烯基上的 $\mathrm{O}$ 配位, 增强探针的荧光. 因此, 探针 14 与 $\mathrm{Hg}^{2+}$ 和 $\mathrm{Fe}^{3+}$ 的结合比分别为 $1: 2$ 和 $1: 3$. NIR 探针 14 对生物 样品的损害小, 组织穿透能力更深, 并能减小背景苂光 和环境诱导的光散射, 具有在活细胞中高灵敏地检测金 属阳离子的潜力.

与上面的反应型不可逆响应机制不同的是, Erdemir 课题组 ${ }^{[51]}$ 通过类似的主客体配位方式成功实现两种金 属离子的检测. 考虑到罗丹明衍生物具有量子产率高、 结构易修饰、吸收和发射波长较长等优点, 可通过醛胺 

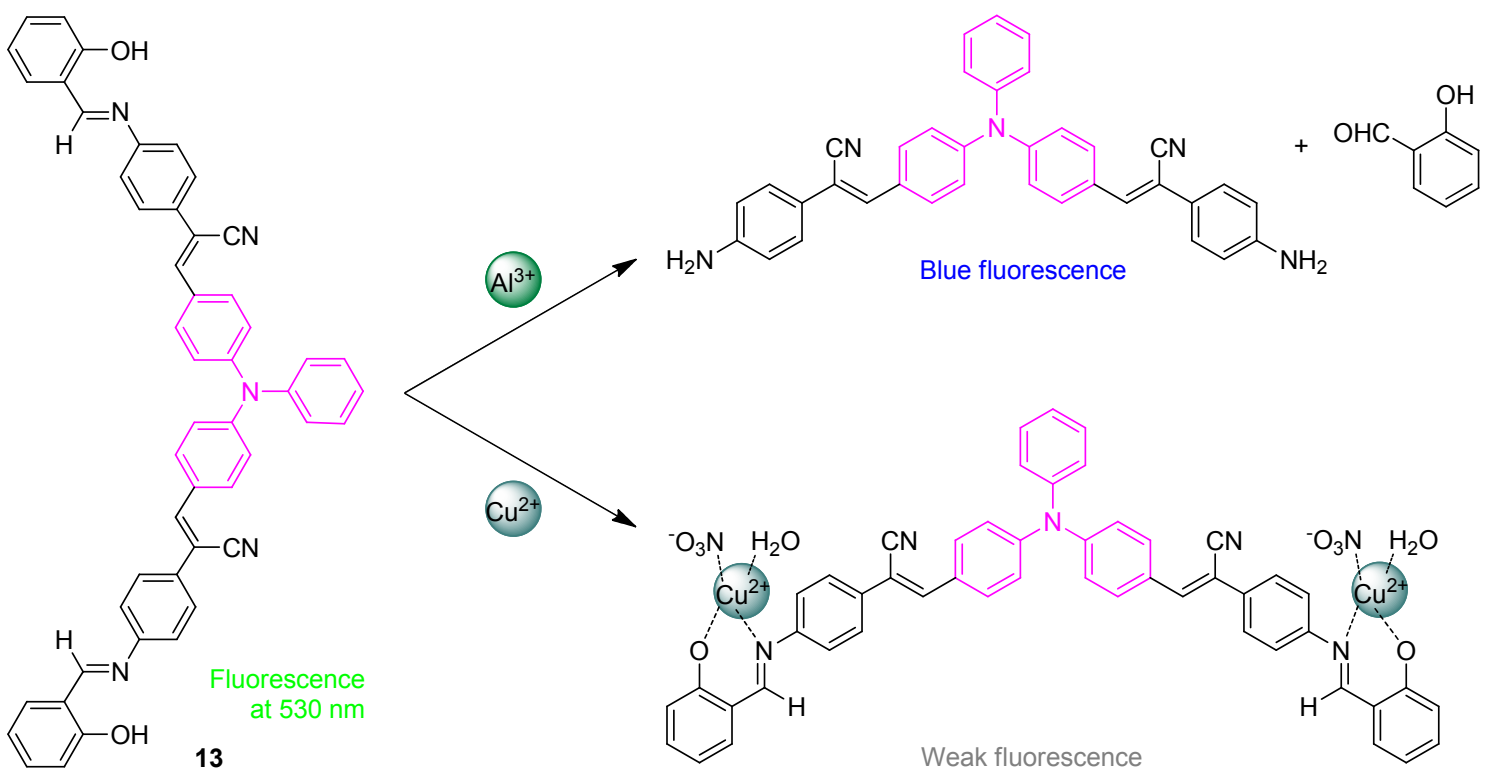

图式 7 探针 $\mathbf{1 3}$ 与 $\mathrm{Al}^{3+}$ 和 $\mathrm{Cu}^{2+}$ 的作用机理

Scheme 7 Sensing mechanism of probe $\mathbf{1 3}$

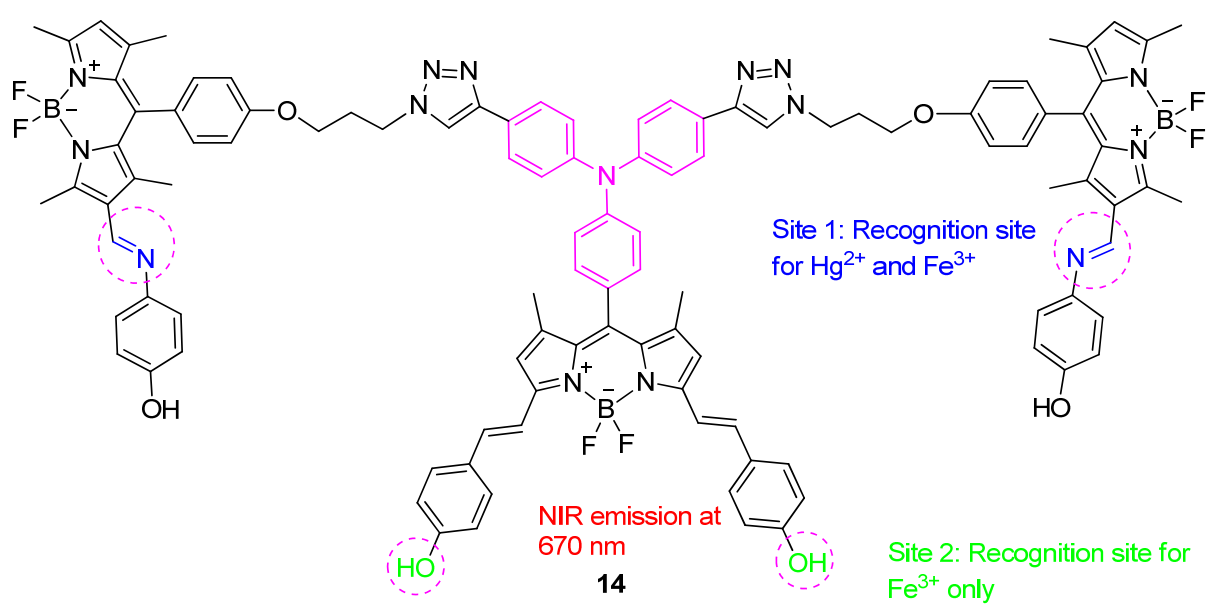

图 2 探针 14 的结构式

Figure 2 Chemical structure of probe 14

缩合反应合成基于三苯胺和罗丹明的探针 15 (Scheme 8). 在 $\mathrm{CH}_{3} \mathrm{CN} / \mathrm{H}_{2} \mathrm{O}(V: V=9: 1)$ 混合溶液中, 探针没有 明显的苂光. 当加入 $\mathrm{Al}^{3+}$ 后, 酚羟基的 $\mathrm{O}$ 、亚胺的 $\mathrm{N}$ 和 罗丹明羰基的 $\mathrm{O}$ 与 $\mathrm{Al}^{3+}$ 以 $1: 1$ 配位后, 探针分子荧光 共振能量转移(FRET)过程被激活, $501 \mathrm{~nm}$ 处的苂光强度 增大, 溶液呈绿色苂光. 当加入 $\mathrm{Hg}^{2+}$ 后, 发生类似的 $1: 1$ 配位并诱导螺内酰胺开环, PET 机制受到抑制, 580 $\mathrm{nm}$ 处的荧光明显增强, 溶液呈橙色荧光. 探针 15 对 $\mathrm{Al}^{3+}$ 和 $\mathrm{Hg}^{2+}$ 具有较高的灵敏度, LOD 低至 $71.8 \mathrm{nmol} / \mathrm{L}$ 和 $0.48 \mu \mathrm{mol} / \mathrm{L}$, 并可制备成测试纸条实现快速检测.

除席夫碱之外, 其他含 $\mathrm{N} 、 \mathrm{~S}$ 原子的五元杂环也常 作为结合位点用于构建三苯胺探针 ${ }^{[52]}$. Easwaramoorthi 课题组 ${ }^{[53]}$ 制备了一种基于富电子三苯胺和缺电子罗丹
宁-3-乙酸的水溶性苂光探针 16 (Scheme 9). 其中, 罗丹 宁-3-乙酸基团负责与金属离子相互作用，并改善探针 在水中的溶解度. 在探针 16 的 $\mathrm{H}_{2} \mathrm{O}$ 溶液中, 由于存在理 想的配位环境, $\mathrm{Ag}^{+}$和 $\mathrm{Hg}^{2+}$ 分别以不同的配位方式与缺 电子的罗丹宁-3-乙酸上的 S、O 原子结合, 溶液荧光由 橙红色分别变为紫色和蓝色. 同时, $\mathrm{Ag}^{+}$和 $\mathrm{Hg}^{2+}$ 分别使 探针 16 的黄色溶液变成紫色和无色溶液, 实现肉眼检 测. 探针 16 的橙红色荧光在 $625 \mathrm{~nm}$ 附近, 处于红外到 近红外(600 900 nm) 区域, 有利于实现 $\mathrm{HaCaT}$ 细胞中 $\mathrm{Ag}^{+}$和 $\mathrm{Hg}^{2+}$ 的无损高灵敏识别.

\section{2 基于三苯胺的阴离子荧光探针}

阴离子在生物体内和工业过程中扮演着不可或缺 


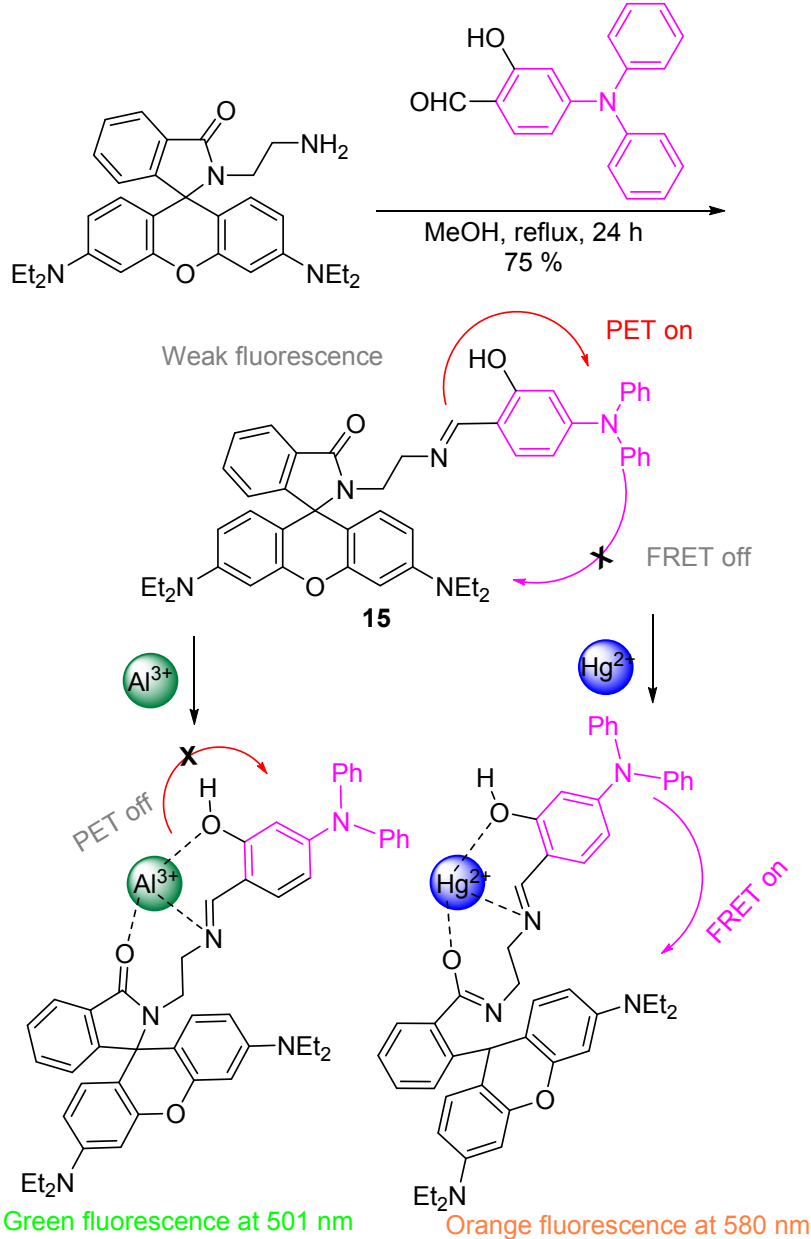

图式 8 探针 15 的合成与作用机理

Scheme 8 Synthesis and sensing mechanism of probe $\mathbf{1 5}$

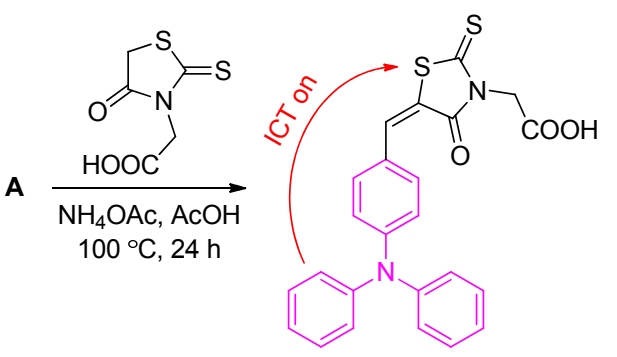

16

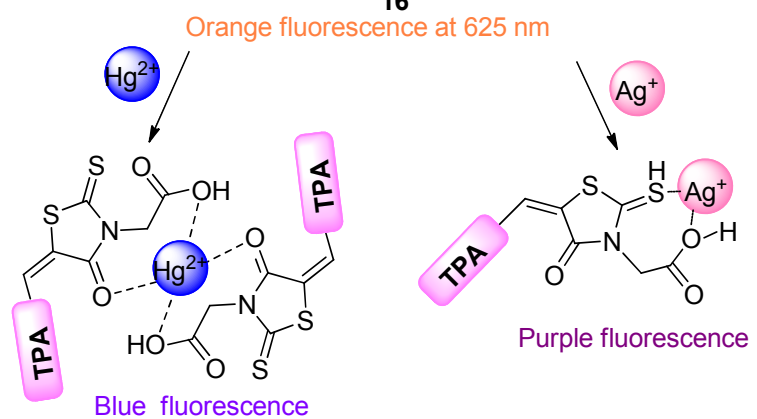

图式 9 探针 16 的合成与作用机理

Scheme 9 Synthesis and sensing mechanism of probe $\mathbf{1 6}$
的角色，检测环境中阴离子污染物具有重要意义 ${ }^{[54]}$. 但 是，阴离子选择性苂光探针的发展稍滞后于阳离子苂光 探针, 因为阴离子具有强的水化作用. 然而, 经过长时 间的研究，阴离子荧光探针和阳离子荧光探针一样获得 长足的发展和进步 ${ }^{[55]}$. 目前, 已经开发了许多用于检测 阴离子的三苯胺苂光探针, 它们主要是利用探针易与分 析物发生化学反应使探针产生荧光变化而实现选择性 传感 ${ }^{[56-57]}$.

在 $\mathrm{CN}^{-}$的识别中，常利用氰化物的特殊亲核性实现 高选择性和高灵敏检测 ${ }^{[58]}$. 叶晓霞课题组 ${ }^{[59]}$ 合成了一 个以三苯胺作为供体、噻吩-苯并三唑-噻吩单元作为 $\pi$ 桥、二氰基乙烯基基团作为受体和识别位点的 D- $\pi-\mathrm{A}$ 分 子 17 (Scheme 10), 其可用作 $\mathrm{CN}^{-}$的比率比色型探针. 在 $\mathrm{THF}$ 水溶液中, 仅有 $\mathrm{CN}^{-}$能与乙烯基发生亲核加成 反应，破坏了探针分子原有的 ICT 机制，在日光灯下溶 液颜色由紫红色变为黄色; 同时, $551 \mathrm{~nm}$ 处的苂光明显 增强, 溶液荧光为橙红色; 而加入其它阴离子, 则没有 明显的颜色变化. 重要的是, 细胞成像实验证明探针 17 可成功应用于生物成像监测嗜铬细胞瘤中的 $\mathrm{CN}^{-}$. 类似 地, 黄晴菲课题组 ${ }^{\left[{ }^{[0]}\right.}$ 也基于三苯胺、噻吩和双氧基乙烯 基骨架，合成了一个 $\mathrm{D}-\pi-\mathrm{A}$ 型荧光探针，能与 $\mathrm{CN}^{-}$发生 亲核加成反应而实现比色和 “turn-on” 响应.

与上面构建的 D- $\pi$-A 型骨架不同的是, 沈永嘉课题 组 ${ }^{[61]}$ 先通过 4-硼酸基三苯胺(B)的 Suzuki 偶联反应引入 了 2 个三苯胺单元作为供体, 再由 Knoevenagel 缩合反 应引入二氯基乙烯基作为识别单元，制备了荧光探针 18 (Scheme 11). 在 $\mathrm{THF} / \mathrm{H}_{2} \mathrm{O}(V: V=99: 1)$ 混合溶液 中, 加入 $\mathrm{CN}^{-}$后，肉眼很容易观察到溶液颜色由紫色变 为黄色, 溶液苂光明显增强为橙红色. 基于 ICT 机理的 比色和荧光双通道探针, 王明峰课题组 ${ }^{[62]}$ 也以三苯胺 单元作为供体进行了类似的研究，构建的 D-A-D 苂光 分子可用于可视化检测 $\mathrm{CN}^{-}$和 $\mathrm{H}_{2} \mathrm{O}$.

$\mathrm{CN}^{-}$除了能与碳碳双键发生加成反应实现苂光检 测外, 碳氧双键 ${ }^{[62]}$ 或者碳氮双键也能作为 $\mathrm{CN}^{-}$的识别 位点. 刘益江课题组 ${ }^{[63]}$ 以三苯胺硼酸衍生物 $\mathbf{B}$ 为原料, 通过 Suzuki 偶联反应合了成近红外苂光探针 19 (Scheme 12), 其中季铵盐部位作为识别位点和吸电子 基团. 在探针 19 的 $\mathrm{CH}_{3} \mathrm{CN}$ 水溶液中, 加入 $\mathrm{CN}^{-}$后, 肉 眼可以很容易观察到溶液颜色由绿色变为黄色; 同时, 发生 “turn-off” 苂光响应. 加入 $\mathrm{CN}^{-}$后的光学变化, 可 能是由探针的共轭结构被破坏以及新形成的加成物在 溶液中聚集所致. 重要的是, 探针 19 不仅能负载在测试 纸条上实现 $\mathrm{CN}^{-}$的实时分析, 而且成功地实现 L929 细 胞中 $\mathrm{CN}^{-}$的检测.

氟离子在牙齿护理和骨质疏松症的治疗中发挥着 


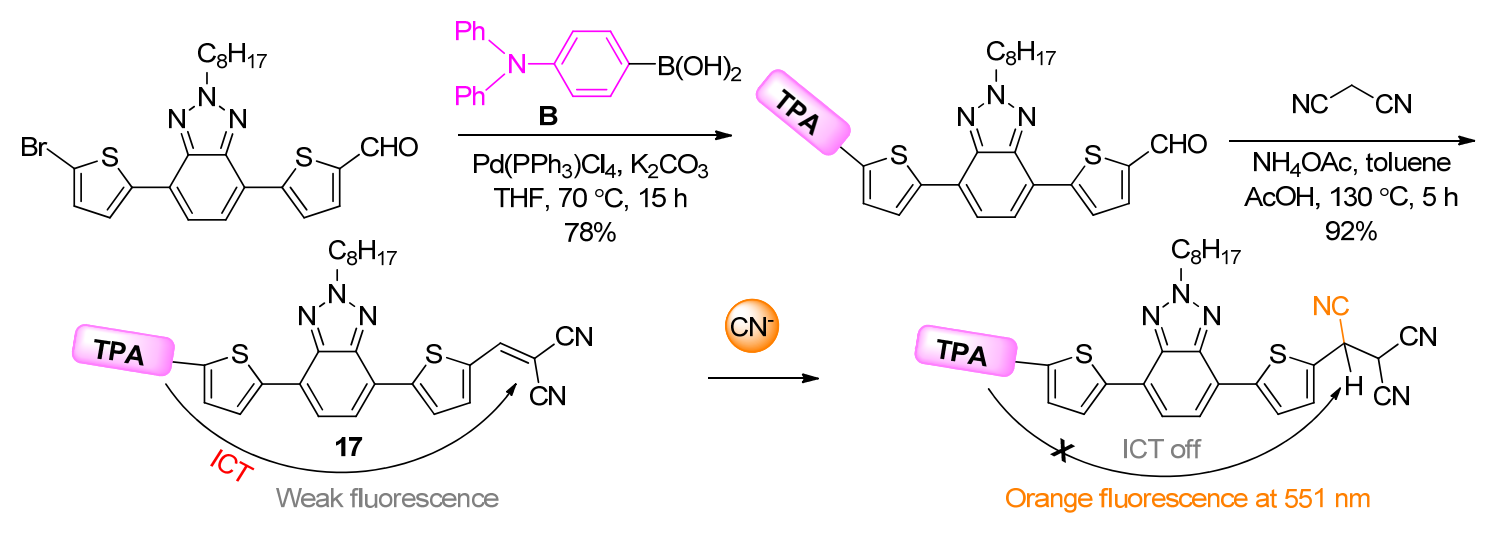

图式 10 探针 17 的合成与作用机理

Scheme 10 Synthesis and sensing mechanism of probe $\mathbf{1 7}$<smiles>O=Cc1cc(Br)c2nsnc2c1Br</smiles>

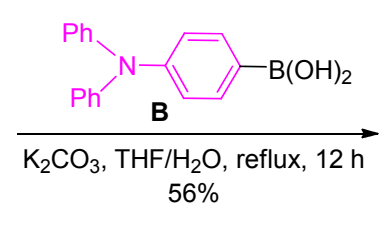
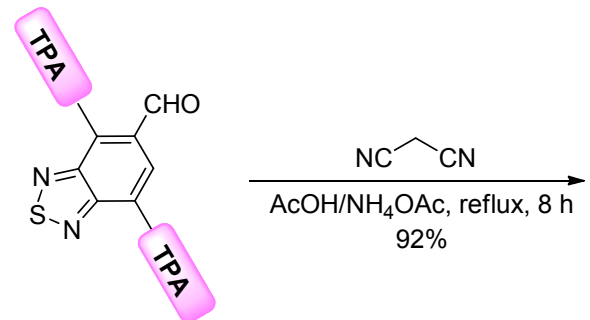<smiles>CC(C)CC1=C(C=C(C#N)C#N)C(CC(C)C)c2nsnc21</smiles>

18

Weak orange-red fluorescence

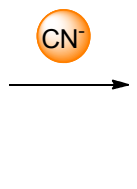

$\mathrm{CN}^{-}$

图式 11 探针 18 的合成与作用机理

Scheme 11 Synthesis and sensing mechanism of probe 18<smiles>CN1/C(=C/C=C2\CCCC(/C=C/C3=[N+](C)c4ccccc4C3(C)C)=C2C=CC2CC2)C(C)(C)c2ccccc21</smiles>

Fluorescence at $780 \mathrm{~nm}$

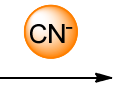

图式 12 探针 19 的作用机理
e 12 Sensing mechanism of probe 19
重要的作用, 但人和动物在大量摄入氟离子时会导致氟 中毒引起肾毒性变化 ${ }^{[64]}$. 因此, 设计合成高选择性的 $\mathrm{F}^{-}$ 荧光探针受到广泛关注 ${ }^{[65]}$. 苏忠民课题组 ${ }^{[66]}$ 通过 Suzuki 偶联反应制备了一种 D-A-D 型的三苯胺基 BODIPY，其具有聚集诱导发射(AIE)和扭曲分子内电荷 转移(TICT)特性, 可作为 $\mathrm{F}^{-}$荧光探针 20 (Scheme 13). 在探针 20 的 $\mathrm{CH}_{3} \mathrm{CN} / \mathrm{H}_{2} \mathrm{O}(\mathrm{V}: V=1: 4)$ 混合溶液中, 溶 液呈红色苂光. 加入 $\mathrm{F}^{-}$后, $\mathrm{F}^{-}$与探针发生亲核加成反应, $\mathrm{B}-\mathrm{N}$ 键断裂, 稳定的二氟嗍桥脱落, 生成新的 $\mathrm{B}-\mathrm{F}$ 和 $\mathrm{N}-\mathrm{H}$ 键. 由于探针 20 的 D-A-D 结构和 TICT 发射被破
坏, 故其红色苂光猝灭. 值得注意的是, 探针 $\mathbf{2 0}$ 已经被 用于活细胞中氟离子荧光成像和选择性检测.

$\mathrm{SO}_{2}$ 是大气中存在的一种主要污染物之一, 长期暴 露于 $\mathrm{SO}_{2}$ 大气中或饮用含高浓度亚硫酸盐 $\left(\mathrm{SO}_{3}^{2-}\right)$ 、亚硫 酸氢盐 $\left(\mathrm{HSO}_{3}^{-}\right)$的水可导致严重的疾病 ${ }^{[67]}$. 因此, 开发 一种检测生物中二氧化硫衍生物的荧光探针具有重要 意义 ${ }^{[68]}$. 孔凡功课题组 ${ }^{[69]}$ 引入罗丹明、三苯胺和乙烯桥, 构建了一个具有多重荧光发射的近红外苂光探针 21 (Scheme 14), 在 PBS/DMSO(二甲基亚砜) $(V: V=9: 1$ ) 混合溶剂中，随着 $\mathrm{SO}_{3}^{2-}$ 加入，探针 21 在 740 和 $810 \mathrm{~nm}$ 


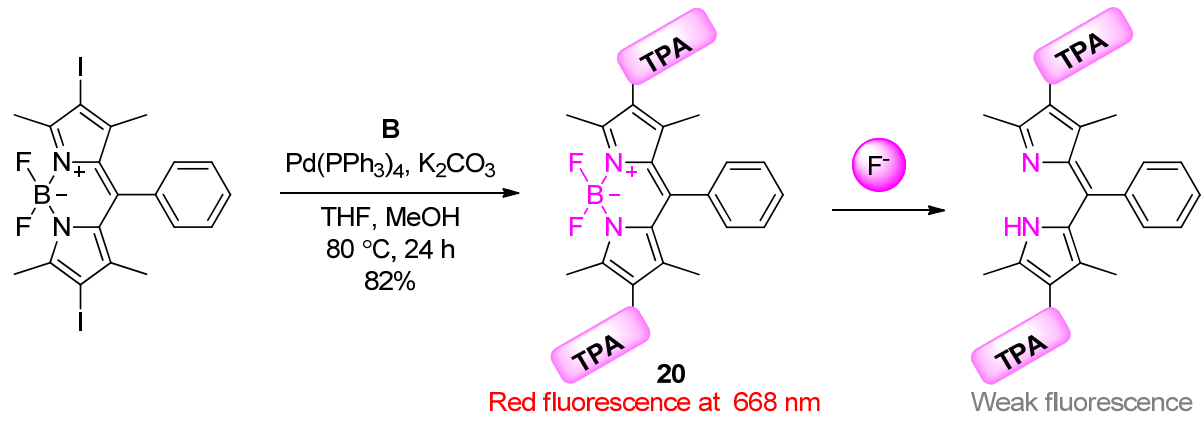

图式 13 探针 20 的合成与作用机理

Scheme 13 Synthesis and sensing mechanism of probe $\mathbf{2 0}$
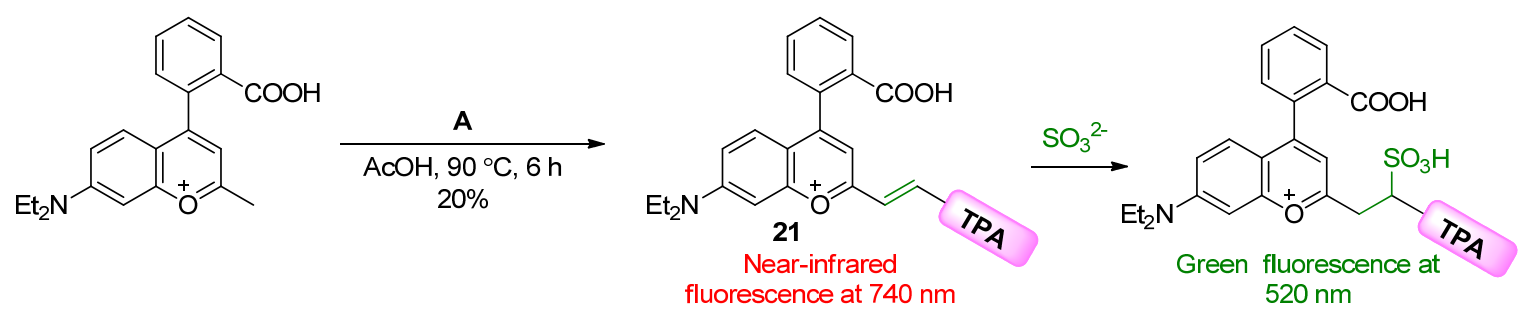

图式 14 探针 21 的合成与作用机理

Scheme 14 Synthesis and sensing mechanism of probe 21

处的发射峰急剧减小; 同时, 在 520 和 $570 \mathrm{~nm}$ 处都可观 察到显著的苂光增强. 比率型探针 21 对 $\mathrm{SO}_{3}^{2-}$ 的 LOD 低至 $3.2 \times 10^{-6} \mathrm{~mol} / \mathrm{L}$. 此外, 该探针还能实现复杂生 物样品(HeLa 细胞和斑马鱼)中 $\mathrm{SO}_{2}$ 衍生物的检测.

活性氧(ROS)在许多生理和病理过程中起着重要作 用. $\mathrm{ClO}^{-}$作为活体中最重要的 $\mathrm{ROS}$ 之一, 在免疫系统抵 抗炎症和维持微生物平衡方面不可或缺, 但 $\mathrm{ClO}^{-}$失衡 会对人体造成不良影响 ${ }^{[70]}$. 鉴于开发活体细胞中的 $\mathrm{ClO}^{-}$ 探针意义重大, 沈健课题组 ${ }^{[71]}$ 以吡啶酰肼和三苯胺衍 生物 $\mathbf{A}$ 为原料合成三苯胺型席夫碱衍生物, 作为 $\mathrm{ClO}^{-}$ 苂光探针 22 (Scheme 15). 最初, 探针 22 在 DMSO/PBS 缓冲液 $(V: V=2: 8)$ 的混合溶液中有强的荧光发射, 加 入 $\mathrm{ClO}^{-}$后 $523 \mathrm{~nm}$ 处的苂光迅速发生明显猝灭, 其他 ROS 没有观察到明显的 “turn-off” 苂光猝灭响应. 这些 独特的变化源于 $\mathrm{ClO}^{-}$的加入触发了氧化过程, 使亚胺 基被转化为醛基. 此外, 荧光成像证明探针 22 具有较好 的生物相容性且能成功在 A549 细胞中灵敏地检测 $\mathrm{ClO}^{-}$.

BODIPY 类染料具有优良的光电性质; 同时, 三苯 胺可以提供重要的空间位阻来防止聚集导致猝灭 (ACQ), 并作为电子供体促进 ICT, 增强 Stokes 位移和 促使苂光红移 ${ }^{[70]}$, 这使三苯胺和 BODIPY 发色团常用于 构建 $\mathrm{ClO}^{-}$苂光探针 ${ }^{[72]}$. 钱鹰课题组 ${ }^{[73]}$ 将 4-乙烯吡啶基 引入三苯胺-BODIPY 醛肟中, 合成了一种新型的 $\mathrm{ClO}^{-}$ 探针 23(图 3). 探针 23 在 THF 水溶液中仅具有极弱的苂 光, 这源于分子内的 $\mathrm{C}=\mathrm{N}$ 异构化. 但探针与 $\mathrm{ClO}^{-}$相互 作用后, 醛肜被氧化成甲醛, 苂光强度显著增强, 量子
产率 $\left(\Phi_{\mathrm{f}}\right)$ 从 0.02 增大到 0.43 , 溶液颜色由粉色变为绿色, 可实现裸眼检测. 探针 $\mathbf{2 3}$ 对 $\mathrm{ClO}^{-}$的 $\mathrm{LOD}$ 为 $7.37 \times 10^{-7}$ $\mathrm{mol} / \mathrm{L}$, 且能在真实水样中高选择性识别. 若将咔唑和 乙烯基呋喃衍生物引入三苯胺-BODIPY 共轭体系中, 可构建 D- $\pi-\mathrm{A}$ 型 ICT 分子 24(图 3), 其亦能用于 $\mathrm{ClO}^{-}$可 视化选择性响应 ${ }^{[74]}$.

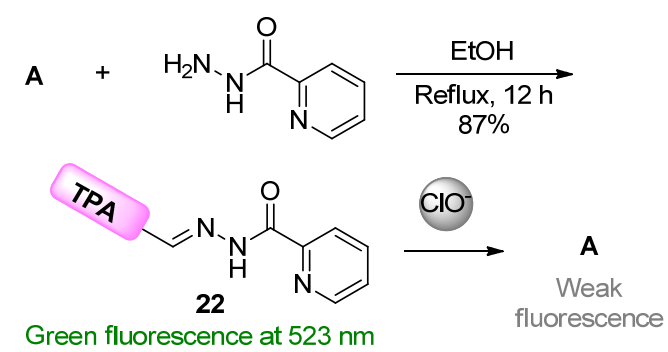

图式 15 探针 22 的合成与作用机理

Scheme 15 Synthesis and sensing mechanism of probe $\mathbf{2 2}$

超氧阴离子 $\left(\mathrm{O}_{2}{ }^{--}\right)$是生命系统中另一种主要的 ROS, 它可作为许多活性氧的前体, 但 $\mathrm{O}_{2}{ }^{-}$过量会导致氧化应 激和氧化还原平衡被破坏, 引起了一系列氧化损伤 ${ }^{[75]}$. 马恒昌课题组 ${ }^{[76]}$ 合成了两个反应型探针 25 和 26 (Scheme 16), 它们分别由富电子三苯胺和二氢吡啶、吡 啶盐活性官能团组成. 在 $\mathrm{O}_{2}{ }^{-}$存在时, 探针 25 和 26 中 的二氢吡啶和吡啶盐单元容易进行脱质子和亲核反应, 致使溶液波长发生红移或蓝移; 同时，苂光强度明显增 强. 因此, 探针 25 和 26 可实现水溶液和 HeLa 细胞中的 $\mathrm{O}_{2}{ }^{-0}$ 高选择性和高灵敏度的双通道识别. 


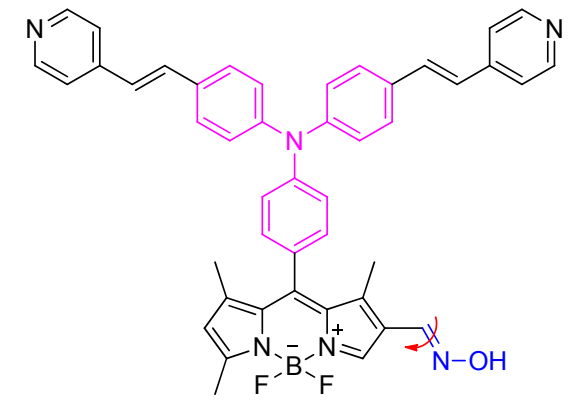

23

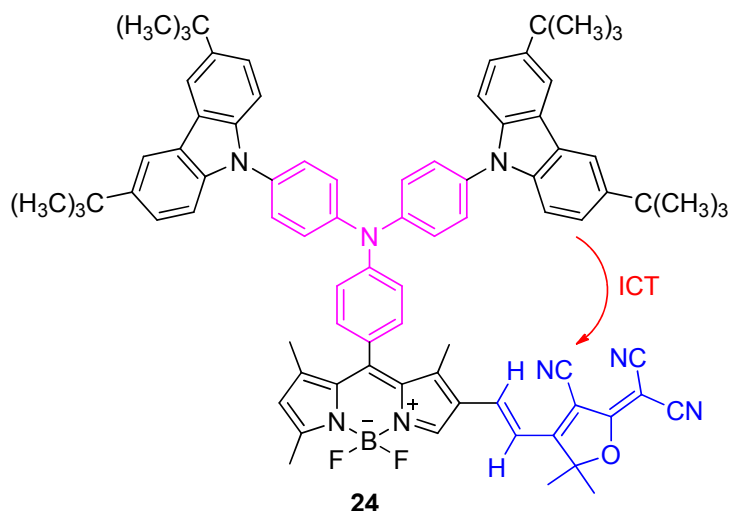

图 3 探针 23 和 24 的分子结构

Figure 3 Molecular structures of probes 23 and 24

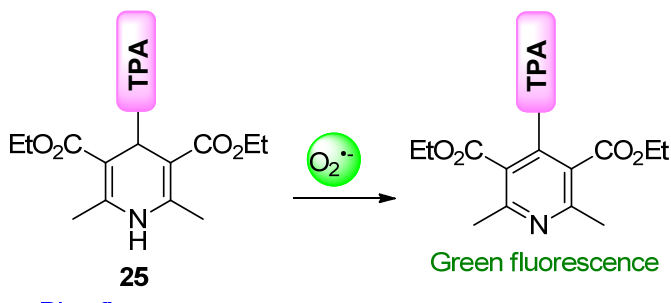

Blue fluorescence

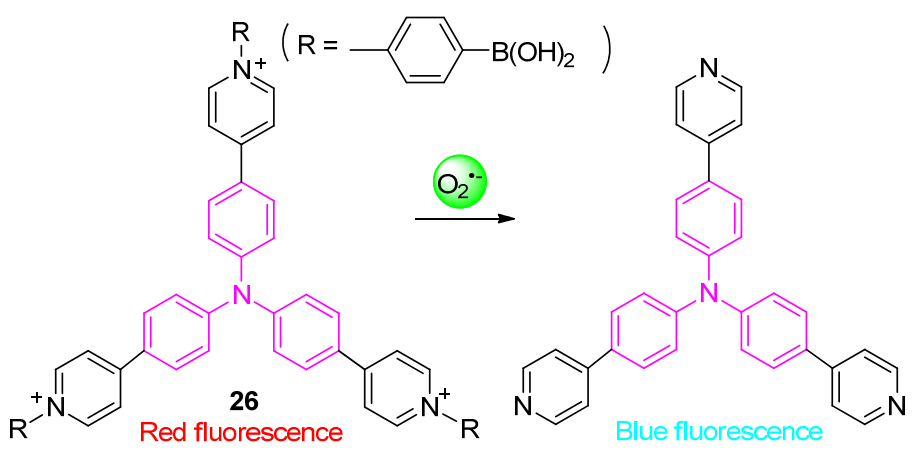

图式 16 探针 25 和 26 的作用机理

Scheme 16 Sensing mechanism of probes 25 and $\mathbf{2 6}$

\section{3 基于三苯胺的中性小分子苂光探针}

\section{1 应用于芳香爆炸物的检测}

环境中的苦味酸(PA)和 2,4,6-三硝基甲苯(TNT)等 硝基芳香爆炸物(NAE)会对公共卫生和安全构成重大威 胁 ${ }^{[77-78]}$. 因此, 发展快速、高选择性的检测痕量 NAE 的 苂光传感器和传感材料具有重要意义 ${ }^{[79]}$. 三苯胺具有 良好的光物理性质和较强的供电子特性 ${ }^{[80]}$, 常用于开 发超灵敏和高选择性的 NAE 苂光探针 ${ }^{[81]}$.

作为一种常见的 NAE, PA 具有极强的爆炸力, 并且 被广泛应用于火箭燃料制造、染料工业和医药工业 ${ }^{[82]}$. 同时, PA 具有较高的水溶性, 是地下水的主要污染物, 摄入过量的 PA 会导致一系列慢性疾病 ${ }^{[83]}$. 因此, 有效 地监测和检测溶液和气相中痕量的 PA 至关重要 ${ }^{[84]}$. 三 苯胺单元的富电子结构和缺电子的 PA 作用, 会导致能 量耗散和苂光猝灭, 从而实现 PA 检测[ ${ }^{[85]}$.

崔月芝课题组 ${ }^{[86]}$ 通过简单的一步反应, 合成了两 个具有 AIE 现象的三苯胺荧光分子 27 和 28 (Scheme 17 ), 并将其发展为高灵敏度的 PA 苂光探针. 在聚集态 $\left(\mathrm{DMSO} / \mathrm{H}_{2} \mathrm{O}, V: V=1: 9\right)$ 下, 探针 27 和 28 水溶液呈红 色荧光, 加入 PA 后荧光强度发生显著猝灭, 但加入其
它 NAE 时荧光强度和颜色无变化. 探针 $\mathbf{2 7}$ 和 $\mathbf{2 8}$ 的猝灭 常数 $\left(K_{\mathrm{sv}}\right)$ 分别为 $1.1 \times 10^{5}$ 和 $5.33 \times 10^{4} \mathrm{~L} \cdot \mathrm{mol}^{-1}$. 相比之 下，探针 27 具有更大的 $K_{\mathrm{sv}}$, 这是由于探针 27 与 $\mathrm{PA}$ 的 LUMO 能极差 $(1.66 \mathrm{eV})$ 大于 $\mathbf{2 8}$ 与 PA 的 LUMO 能极差 $(1.15 \mathrm{eV})$, 因而具有较强的 PET 驱动力导致的.

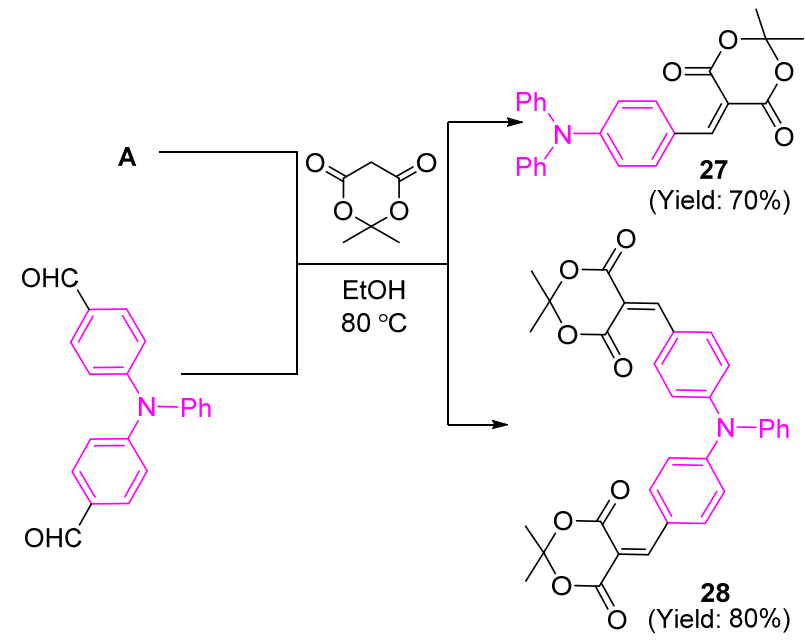

图式 17 探针 $\mathbf{2 7}$ 和 $\mathbf{2 8}$ 的合成路线

Scheme 17 Synthesis route of probes 27 and 28

TNT 是另一种极强的 NAE，始见于第一次世界大 
战, 其痕量的检测是一个全球关注的问题, 对国土安 全、反恐、军事问题和环境保护都至关重要 ${ }^{[87]}$. 王金亮 课题组 ${ }^{[88]}$ 通过 Corey-Fuchs 反应和偶联反应在二噻吩核 外围修饰不同数量的三苯胺基(TPA), 分别合成了三个 AIE 苂光分子 29 31 (图 4). 富电性的分子 29 和 31 在 聚集状态下 ( $99 \%$ 含水率)能有效发射, 并能够发展为 TNT 荧光探针. 在混合溶剂体系中加入 $80 \mu \mathrm{mol} / \mathrm{L}$ 的 TNT 时, 其对探针 29 和 31 的猝灭效率分别为 $55 \%$ 和 $61 \%$, 并进一步计算得出 $K_{\mathrm{sv}}$ 分别为 $0.69 \times 10^{5}$ 和 $0.75 \times 10^{5} \mathrm{~L} \cdot \mathrm{mol}^{-1}$. 另外, 探针 29 和 31 还能选择性检测 一些硝基脂肪族爆炸物, 如 3-硝基 1,2,4-三唑-5-酮、环 四亚甲基四硝胺和六硝基六氮杂异伍兹烷.

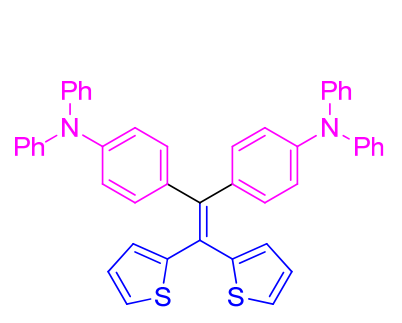

29

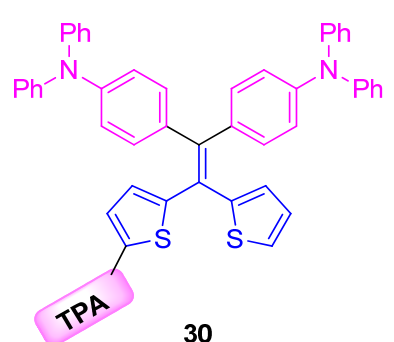

30

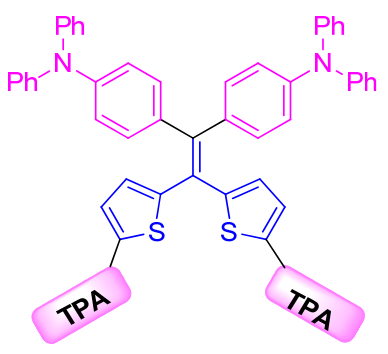

31

图 4 探针 29 31 的分子结构

Figure 4 Molecular structures of probes 29 31

六元含氮杂环具有良好的光学性质和潜在的作用 位点 ${ }^{[89-90]}$, 也常被用于爆炸物探针, 包括与三苯胺的组 合, 体现出多功能探针检测的简便和高效特性. Verbitskiy 课题组 ${ }^{[91]}$ 以苯并吡嗪或吡嗪作为受体, 噻吩作为 $\pi$ 桥, TPA 作为供体, 设计合成了一系列 D- $\pi$-A 苂光分子 (Eq. 2), 一方面, 探针 32 35 能够在二氯甲烷溶液和气 相中与多种 NAE 发生 PET 作用导致荧光发生显著猝灭, 成功实现硝基苯、PA 以及 TNT 等的灵敏识别. 其中, $\mathbf{3 4}$ 对 NAE 显示出最高的 $K_{\mathrm{sv}}$ 和最低的 LOD, 这与 34 具有 较大的量子产率是一致的. 另一方面, 探针 32 35 与二 乙胺和三乙胺蒸汽作用后, 苂光强度明显增大, 这表明 $32 \sim 35$ 也可用于气相脂肪族胺的实时检测.

\section{2 应用于活性硫的检测}

细胞内生物硫醇如半胱氨酸(Cys)、同型半胱氨酸 (Hcy)和谷胱甘肽 $(\mathrm{GSH})$ 在生物系统中起着重要作用 ${ }^{[92]}$.
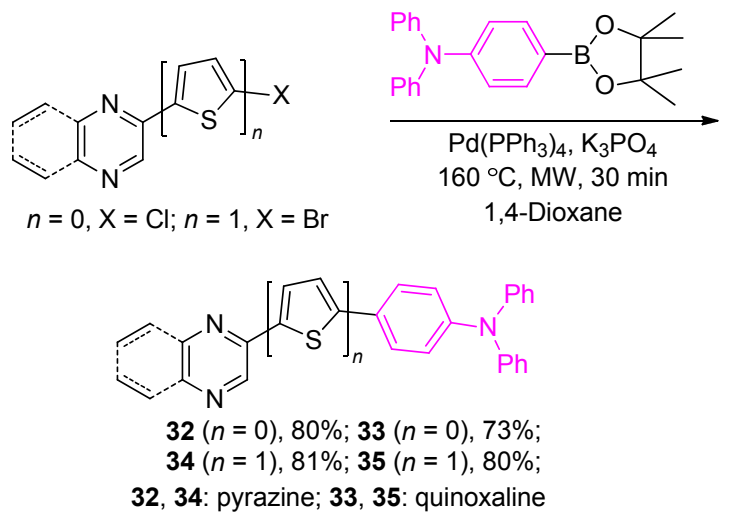

它们的异常水平会导致许多疾病, 如肝损伤、白细胞减 少、癌症和艾滋病等 ${ }^{[93]}$. 因此, 对生物样品中含硫生物 分子的检测就显得尤为重要. 周虹屏课题组 ${ }^{[94]}$ 基于三 苯胺骨架合成了 4 个 D- $\pi$-A 苂光探针 36 39 (Scheme 18), 它们可通过两种不同的作用机制选择性识别 GSH. 其中, 探针 36 和 37 与 GSH 作用诱导亚胺键发生水解, 苂光强度逐渐增强, 该 GSH 直接检测中 LOD 分别

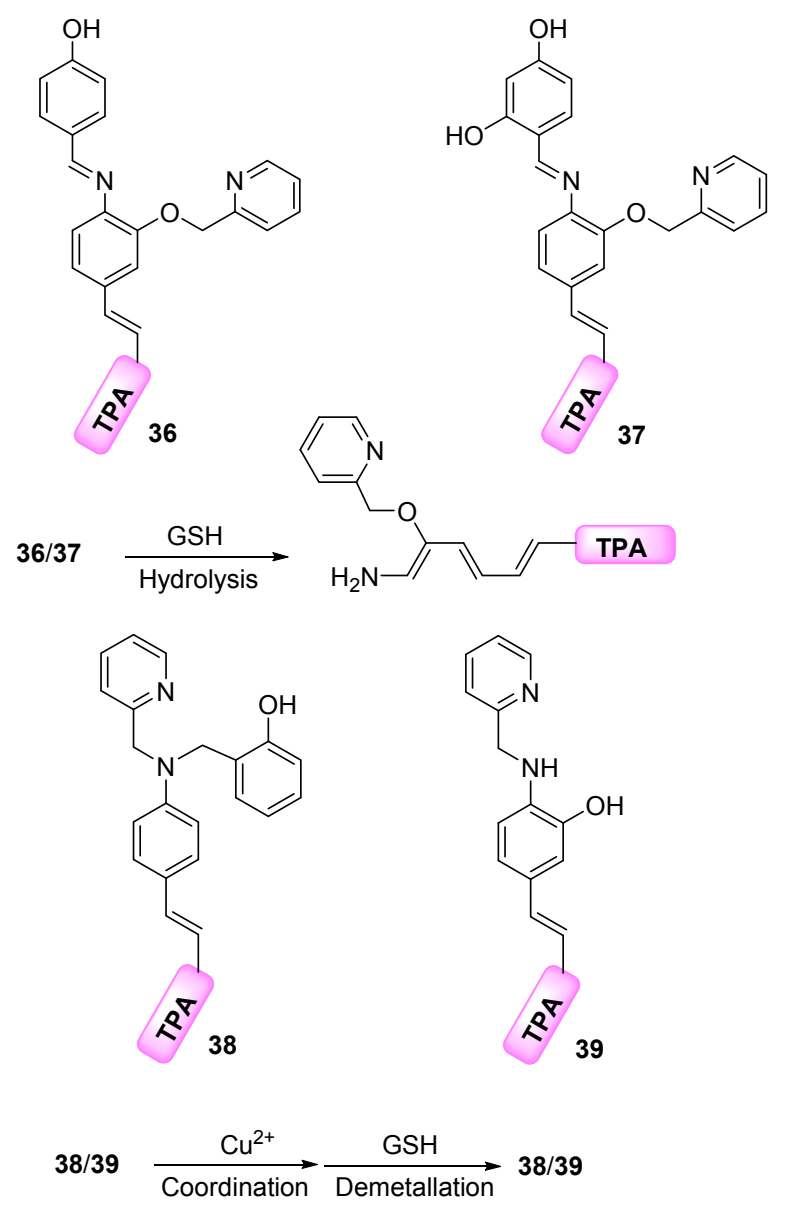

图式 18 探针 36 39 的分子结构和作用机理 Scheme 18 Molecular structures and sensing mechanism of probes $36 \sim 39$ 
为 0.106 和 $0.131 \mu \mathrm{mol} / \mathrm{L}$; 探针 38 和 39 先与 $\mathrm{Cu}^{2+}$ 配位, 所得有机金属配合物与 GSH 发生原位置换反应, 脱去 $\mathrm{Cu}^{2+}$ 的同时释放探针分子, 荧光发生 “on-off-on” 型响 应, 对 GSH 的间接检测的 LOD 分别为 0.394 和 0.977 $\mathrm{nmol} / \mathrm{L}$. 不仅如此, 探针 36 39 还都可实现 HeLa 细胞 内 GSH 的检测.

苯硫酚 $(\mathrm{PhSH})$ 是另一种活性硫物种(RSS), 作为杀 虫剂被广泛用于化学工业中. 但苯硫酚也是一种高污染 物和有毒化合物, 长期接触暴露在空气或水中的硫酚可 导致全身损伤, 故其灵敏检测对环境和人体健康具有重 要意义 ${ }^{[95]}$. 最近, 李俊叶课题组 ${ }^{[96]}$ 基于三苯胺荧光团合 成一个新型 ICT 荧光分子, 并将其发展为 $\mathrm{PhSH}$ 探针 40 (Scheme 19). 在探针 $\mathbf{4 0}$ 的 DMSO/PBS $(V: V=5: 5)$ 缓 冲溶液中加入 $\mathrm{PhSH}$, 其进攻二硝基苯醚键发生取代反 应, 随着硫醚的离去, 溶液苂光变为蓝绿色, 荧光强度 发生肉眼可见的增强, 响应时间小于 $15 \mathrm{~s}$. 在 $\mathrm{pH}$ 值为 $4.0 \sim 12.0$ 范围内的溶液中, 探针 40 荧光强度基本不变, 表现出良好的光和化学稳定性, 能够扩展到实际水样和
活细胞中 $\mathrm{PhSH}$ 的检测, 具有潜在的应用价值.

硫化氢 $\left(\mathrm{H}_{2} \mathrm{~S}\right)$ 是 RSS 物种中最小的成员，同时，作为 第三内源性气体信号化合物, 在调节生理过程起着重要 的作用, 其水平异常与许多疾病相关 ${ }^{[97]}$. 鉴于 $\mathrm{H}_{2} \mathrm{~S}$ 在生 物学中检测的重要性, 吴水珠课题组 ${ }^{[88]}$ 也基于二硝基 苯醚识别位点, 以三苯胺和喹啉为苂光团, 设计合成了 一个 $\mathrm{H}_{2} \mathrm{~S}$ 荧光探针 41 (Scheme 20). 由于具有强吸电子 能力的二硝基苯基团通过 PET 机制猝灭分子苂光, 探针 41 在 $\mathrm{PBS} / \mathrm{DMSO}(V: V=99: 1)$ 缓冲溶液中没有明显 荧光. 当 $\mathrm{H}_{2} \mathrm{~S}$ 与探针作用后, 触发裂解反应, 二硝基苯 基团的猝灭作用消失，不良溶剂中的疏水性增加，产生 AIE 效应，观察到一个明显的 “turn-on” 苂光信号. 此 外，探针 41 不仅能成功用于监测食品样品中的 $\mathrm{H}_{2} \mathrm{~S}$, 以 区分食品变质的程度, 还能监测活细胞中 $\mathrm{H}_{2} \mathrm{~S}$ 浓度的变 化以及进行 HCT-116 移植瘤组织内的内源性 $\mathrm{H}_{2} \mathrm{~S}$ 成像. 类似地, 黄祖胜课题组 ${ }^{[99]}$ 以 TPA 和 BODIPY 为荧光团, 以 2,4-二硝基磺酰为反应位点, 合成了相似作用机理的 $\mathrm{H}_{2} \mathrm{~S}$ 荧光探针.
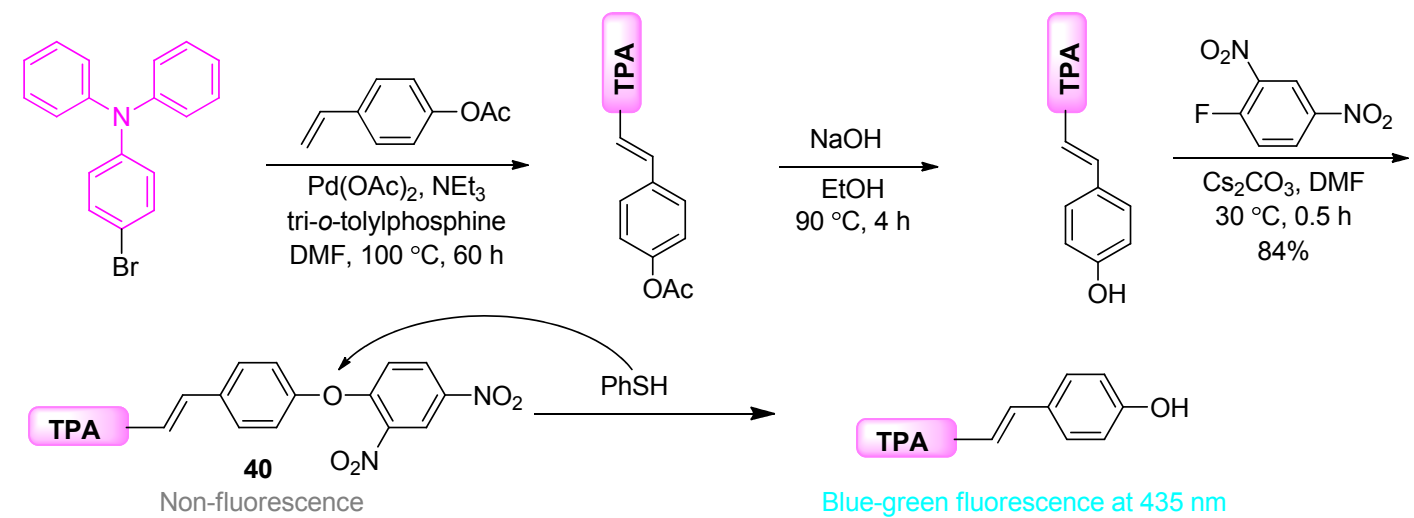

Blue-green fluorescence at $435 \mathrm{~nm}$

图式 19 探针 40 的合成与作用机理

Scheme 19 Synthesis and sensing mechanism of probe $\mathbf{4 0}$

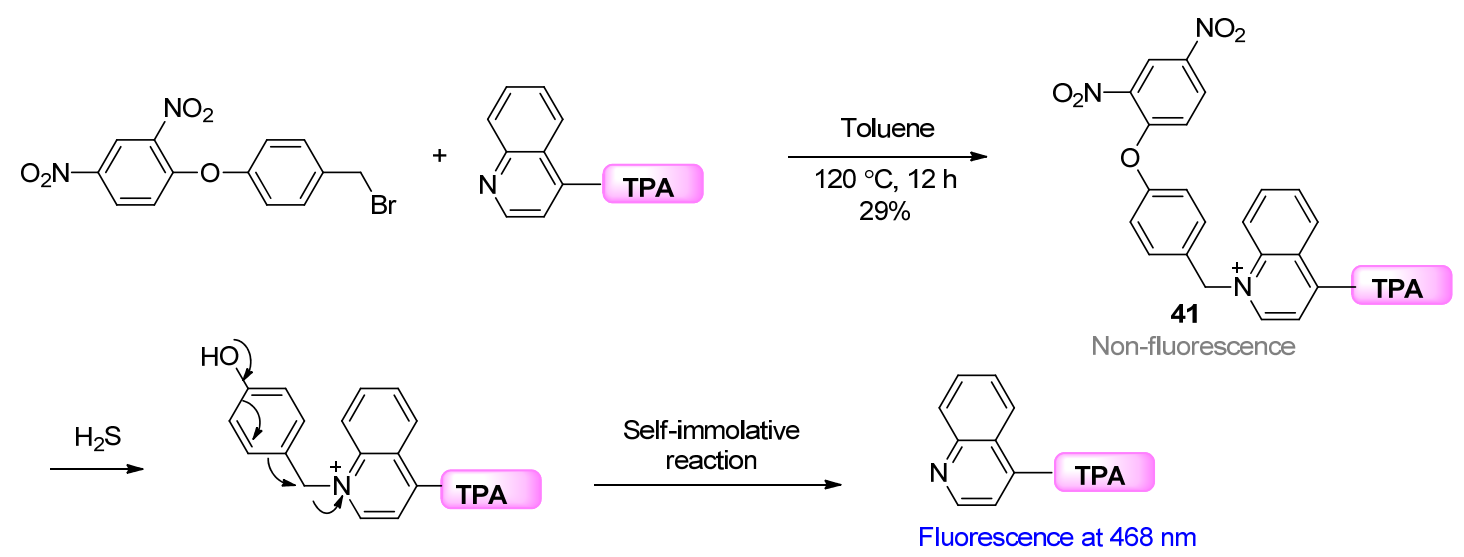

图式 20 探针 41 的合成与作用机理

Scheme 20 Synthesis and sensing mechanism of probe 41 


\section{3 应用于其他中性小分子的检测}

除了硝基爆炸物、RSS 等中性小分子的检测对自然 环境和生物体健康具有重要意义之外, 还有一些中性小 分子的高灵敏性识别也同样受到关注. 马恒昌课题 组 ${ }^{[100]}$ 以 TPA 为核心, 触手状的甲基丙烯酸酯为支链, 合成了一个水溶性 D-A 型苂光探针 42(图 5), 可用于日 光灯和紫外灯下可视化检测赖氨酸(Lys), 42 对 Lys 的特 异性识别是通过作用后形成亚胺导致荧光增强而实现 的.

Mondal 课题组 ${ }^{[101]}$ 以三苯胺为供体, 苯并咪唑苂光 团为受体, 碳碳双键和苯环为 $\pi$ 桥设计合成了一个新型 ICT 苂光分子 43(图 5), 并将其开发为有机磷神经毒剂 氯磷酸二乙酯(DCP)比色型苂光探针. 在探针溶液中加 入 DCP 后, 溶液颜色由无色变为黄色, 荧光由青色变成 深黄色. 这种明显的比色型变化源于三苯胺上的氮原子 可加速共轭, 导致苯并咪唑上的氮原子很容易地与 $\mathrm{DCP}$ 上的亲电中心 $\mathrm{P}$ 原子)发生亲核取代反应, 并最终 提高分子内 ICT 效率. 重要的是, 探针 43 还能发展为一 种便携式装置检测气态和液态 DCP, 具有实时监测的实 际应用价值.

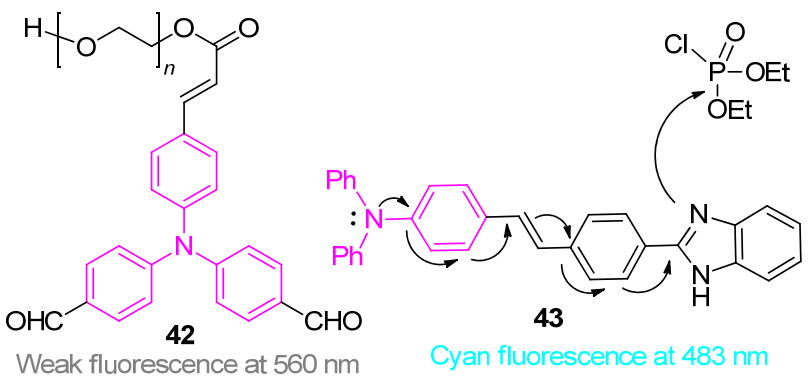

图 5 探针 42 和 43 的分子结构

Figure 5 Molecular structures of probes $\mathbf{4 2}$ and $\mathbf{4 3}$

具有 AIE 性能的三苯胺探针可用于高选择性地检 测食品 ${ }^{[102]}$ 和环境中的中性有害物. 王新潮课题组 ${ }^{[103]}$ 以 三苯胺氨基衍生物和邻氨基苯甲醛为原料, 合成了一种 新型的双席夫碱苂光探针 $\mathbf{4 4}$ (Scheme 21), 用于检测危险 化学品 $\mathrm{N}_{2} \mathrm{H}_{4}$. 探针 44 在 $N, N$-二甲基甲酰胺(DMF)/ $\mathrm{H}_{2} \mathrm{O}$ 混合体系中表现出较强的 AIE 特性, 含水率高时溶液呈 亮黄色苂光 $\left(\lambda_{\mathrm{em}, \max }=560 \mathrm{~nm}\right)$. 当加入 $\mathrm{N}_{2} \mathrm{H}_{4}$ 后, 由于特殊 的还原反应, 探针分子的 $\mathrm{CH}=\mathrm{N}$ 键转变为 $\mathrm{CH}_{2}-\mathrm{NH}$ 键, 分子的水溶性增加, AIE 减弱; 同时, ESIPT 机制受到抑 制, 故溶液荧光发生猝灭. 传感实验表明, 探针 44 对检 测 $\mathrm{N}_{2} \mathrm{H}_{4}$ 的灵敏度高, 检出限低至 $55.1 \mathrm{nmol} / \mathrm{L}$, 且适用 于外源 $\mathrm{N}_{2} \mathrm{H}_{4}$ 在 $\mathrm{HeLa}$ 细胞中的苂光成像.

一些溶剂小分子的检测也是个令人感兴趣的话题, 包括水分含量的变化 ${ }^{[79,104]}$, 也可通过三苯胺基苂光探

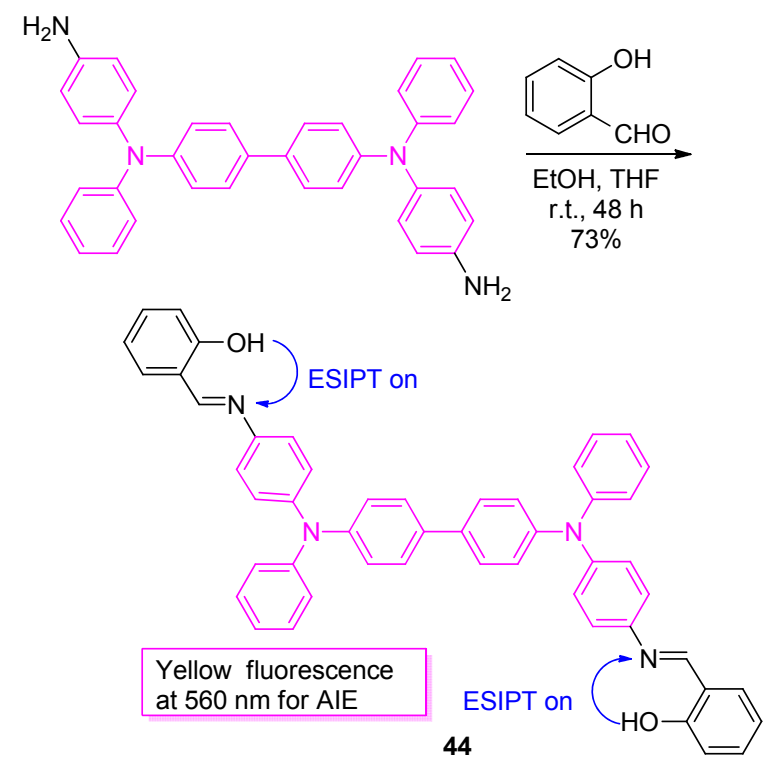

图式 21 探针 44 的合成与作用机理

Scheme 21 Synthesis and sensing mechanism of probe 44

针实现. 阮银兰课题组 ${ }^{[105]}$ 将三苯胺-四苯基吡嗪发展为 苂光探针 45(图 6), 用于检测有机溶剂中的痕量水. 随 着体系中水含量的增大 $(0 \sim 60 \%)$, 苂光发射峰发生红 移，这是因为在高极性水的存在下，分子的 D-A 偶极子 强度发生改变，影响电子受体区域的成键，引起苂光强 度和苂光峰波长的变化.

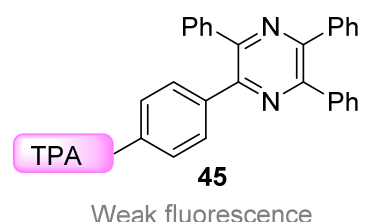

图 6 探针 45 和 46 的分子结构

Figure 6 Molecular structures of probes 45 and 46

除水外，有机溶剂的检测也能通过三苯胺探针实 现. 王勇课题组 ${ }^{[106]}$ 以 4-乙炔基三苯胺和 4-甲基苯磺酰 基叠氮化物为原料，通过 Click 反应合成 D- $\pi$-A 型苂光 探针 46(图 6), 用于检测甲醇. 探针 46 在甲醇溶剂中, 蓝色苂光明显增强，而在正己烷、四氢呋喃、乙醇和二 甲基亚砜等不同极性溶剂中苂光均没有增强. 所以，探 针 46 能够特异性识别 $\mathrm{CH}_{3} \mathrm{OH}$, 且 LOD 小于 $0.7 \%$ (体积 分数). 这是因为探针扭曲的构象、共轭 $\mathrm{D}-\pi-\mathrm{A}$ 结构以及 氢键和溶剂极性协同作用，共同促进了特异性荧光响 应. 有趣的是, 探针在 $\mathrm{MeOH} / \mathrm{H}_{2} \mathrm{O}(V: V=3: 1)$ 混合溶 剂中还能通过苂光猝灭检测铜离子.

\section{4 结束语}

综上所述，由于在溶液中具有多样的发光特性以及 良好的光稳定性和化学稳定性, 近年来功能化的三苯胺 
苂光探针已经获得较好的发展. 在分子设计方面, 三苯 胺骨架可作为电子供体位于分子的两端，构建 D-A、

$D-\pi-A 、 D-A-D$ 型探针, 或者是作为荧光团和连接桥与 识别受体单元共同构建探针. 在检测对象方面, 三苯胺 苂光探针能够识别各式各样的分析物, 包括阳离子 (如 $\mathrm{Al}^{3+} 、 \mathrm{Cu}^{2+} 、 \mathrm{Fe}^{3+} 、 \mathrm{Zn}^{2+} 、 \mathrm{Hg}^{2+} 、 \mathrm{Ag}^{+}$等)、阴离子(如 $\mathrm{CN}^{-} 、 \mathrm{~F}^{-} 、 \mathrm{SO}_{3}^{2-} 、 \mathrm{ClO}^{-} 、 \mathrm{O}_{2}{ }^{-}$等)和中性小分子(如 NAE、 RSS、 $\mathrm{N}_{2} \mathrm{H}_{4} 、 \mathrm{H}_{2} \mathrm{O}$ 等). 在检测机理方面, 三苯胺探针能 根据探针结构和检测对象巧妙地利用不同的作用机理 (如 ICT、PET、ESIPT、FRET、CHEF、CHEQ)实现高 选择性和高灵敏度识别.

虽然三苯胺苂光探针在分子设计和检测应用上已 有丰富的研究, 但随着社会的发展, 监管机构对生物和 环境分析的要求越来越严格, 现有的探针可能达不到在 实际应用中所需的选择性或灵敏度. 因此, 我们需要通 过简单绿色的合成方法在带有反应性官能团(如醛基、

嗍酸基、氨基、卤素、炔基)的三苯胺骨架上桥接上新 的受体或发光团, 使其具有特异性的识别位点和合理的 作用机制，特别是构建近红外发光和高量子效率的 AIE 荧光探针, 从而实现复杂环境中的实际样品检测和生物 体内成像.

\section{References}

[1] Murakami, T. N.; Koumura, N. Adv. Energy Mater. 2019, 9, 1802967.

[2] Rodriguez-Seco, C.; Mendez, M.; Roldan-Carmona, C.; Pudi, R.; Nazeeruddin, M. K.; Palomares, E. J. Angew. Chem., Int. Ed. 2020, $13,5303$.

[3] Li, Y. Y.; Liu, S. J.; Ni, H. W.; Zhang, H. K.; Zhang, H. Q.; Chuah, C.; Ma, C.; Wong, K. S.; Lam, J. W. Y.; Kwok, R. T. K.; Qian, J.; Lu, X. F.; Tang, B. Z. Angew. Chem., Int. Ed. 2020, 59, 12822.

[4] Xu, W. H.; Wang, D.; Tang, B. Z. Angew. Chem., Int. Ed. 2021, DOI: 10.1002/anie.202005899.

[5] Jiang, M. J.; Gu, X. G.; Lam, J. W. Y.; Zhang, Y. L.; Kwok, R. T. K.; Wong, K. S.; Tang, B. Z. Chem. Sci. 2017, 8, 5440.

[6] Shen, P.; Hua, J. Y.; Jin, H. D.; Du, J. Y.; Liu, C. L.; Yang, W.; Gao, Q. Y.; Luo, H. J.; Liu, Y.; Yang, C. Y. Sens. Actuators, B 2017, 247, 587.

[7] Li, Y.; Gu, Z. Y.; He, T.; Yuan, X. C.; Zhang, Y. Y.; Xu, Z.; Qiu, H. Y.; Zhang, Q.; Yin, S. C. Dyes Pigm. 2020, 173, 107969.

[8] Yang, X. D.; Chen, X. L.; Lu, X. D.; Yan, C. G.; Xu, Y. K.; Hang, X. D.; Qu, J. Q.; Liu, R. Y. J. Mater. Chem. C 2016, 4, 383.

[9] Xia, Y.; Zhang, H. H.; Zhu, X. J.; Zhang, G. J.; Yang, X. Y.; Li, F.; Zhang, X. J.; Fang, M.; Yu, J. H.; Zhou, H. P. Dyes Pigm. 2018, $155,159$.

[10] Abdurahman, A.; Wang, L.; Zhang, Z. X.; Feng, Y. T.; Zhao, Y. H.; Zhang, M. Dyes Pigm. 2020, 174, 108050.

[11] Cai, W. N.; Xiao, T. D.; Niu, H. J.; Bai, X. D.; Zhang, Y. H.; Wang, C.; Wang, W.; Qi, H. Sens. Actuators, B 2017, 252, 330.

[12] Wu, X. M.; Mao, Y.; Wang, D. Y.; Huang, Q.; Yin, Q. X.; Zheng, M.; Hu, Q. H.; Wang, H. Q. Sens. Actuators, B 2020, 307, 127681.

[13] Czarnik, A. W. Fluorescent Chemosensors for Ion and Molecule Recognition, American Chemical Society, Washington, DC, 1993.

[14] Pang, C.-M.; Luo, S.-H.; Jiang, K.; Wang, B.-W.; Chen, S.-H.; Wang, N.; Wang, Z.-Y. Dyes Pigm. 2019, 170, 107651.

[15] Li, H.; Zhang, R. L.; Li, C. X.; Huang, B.; Yu, T. T.; Huang, X. D.; Zhang, X. J.; Li, F.; Zhou, H. P.; Tian, Y. P. Org. Biomol. Chem.
2017, 15, 598.

[16] Gao, Z.-Y.; Zhang, C.-J.; Zhang, X.; Xing, S.; Yao, J.-S.; Qiao, C.-D.; Liu, W.-L. Appl. Sci. 2019, 9, 577.

[17] Mu, Y.-L.; Zhang, C.-J.; Gao, Z.-L.; Zhang, X.; Lu, Q.; Yao, J.-S.; Xing, S. Synth. Met. 2020, 262, 116334.

[18] Gan, X. P.; Li, W.; Li, C. X.; Wu, Z. C.; Liu, D.; Huang, B.; Zhou, H. P.; Tian, Y. P. Sens. Actuators, B 2017, 239, 642.

[19] Zhang, H. H.; Wei, Z. Y.; Xia, Y.; Fang, M.; Zhu, W. J.; Yang, X. Y.; Li, F.; Tian, Y. P.; Zhang, X. J.; Zhou, H. P. Spectrochim. Acta, Part A 2018, 196, 256.

[20] Li, W.; Gan, X. P.; Liu, D.; Tian, X. H.; Yu, J. H.; Tian, Y. P.; Wu, J. Y.; Zhou, H. P. RSC Adv. 2016, 6, 44599

[21] Shi, F.; Cui, S. Q.; Liu, H. L.; Pu, S. Z. Dyes Pigm. 2020, 173 107914.

[22] Chen, H.; Yang, P.; Li, Y. H.; Zhang, L. L.; Ding, F.; He, X. J.; Shen, J. L. Spectrochim. Acta, Part A 2020, 224, 117384.

[23] Jiang, K.; Wu, Y.-C.; Wu, H.-Q.; Li, S.-L.; Luo, S.-H.; Wang, Z.-Y. J. Photochem. Photobiol., A 2018, 350, 52.

[24] Huang, Q. X.; Peng, Z. X.; Xie, X. R.; Tang, Z. F.; Lei, M. ChemistrySelect 2019, 4, 13490.

[25] Pan, C. Q.; Wang, K.; Ji, S. M.; Wang, H. Q.; Li, Z. Z.; He, H. H.; Huo, Y. P. RSC $A d v$. 2017, 7, 36007.

[26] Zhang, Z. X.; Li, F.; He, C. Y.; Ma, H. W.; Feng, Y. T.; Zhang, Y. N.; Zhang, M. Sens. Actuators, B 2018, 255, 1878.

[27] Jiang, K.; Chen, S.-H.; Luo, S.-H.; Pang, C.-M.; Wu, X.-Y.; Wang, Z.-Y. Dyes Pigm. 2019, 167, 164.

[28] Wei, W. G.; Jin, Y.; Han, T.; Du, B.; Zhi, X. J.; Wei, C. J.; Yuan, S. C. Aust. J. Chem. 2018, 77, 890.

[29] Liu, B.; Tan, Y. H.; Hu, Q. H.; Wang, Y. Y.; Wu, X. M.; Huang, Q. X.; Zhang, W. F.; Zheng, M.; Wang, H. Q. Chem. Pap. 2019, 73, 3123 .

[30] Shi, W.; Zhao, S. Y.; Su, Y.; Hui, Y. H.; Xie, Z. F. New J. Chem. 2016, 40, 7814.

[31] Zhang, Q. S.; Li, Y. H.; Zuo, H. J.; Wang, C. Y.; Shen, Y. J. J. Photochem. Photobiol., A 2017, 332, 293

[32] Zhang, Q. S.; Zhang, J.; Zuo, H. J.; Wang, C. Y.; Shen, Y. J. Tetrahedron 2017, 73, 2824

[33] Xiao, H. B.; Zhang, Y. Z.; Zhang, W.; Li, S. Z.; Tan, J. J.; Han, Z. Y. Mater. Chem. Phys. 2017, 192, 268.

[34] Xiao, H. B.; Zhang, Y. Z.; Li, S. Z.; Zhang, W.; Han, Z. Y.; Tan, J. J.; Zhang, S. Y.; Du, J. Y. Sens. Actuators, B 2016, 236, 233.

[35] Wu, Y.-C.; Jiang, K.; Luo, S.-H.; Cao, L.; Wu, H.-Q.; Wang, Z.-Y. Spectrochim. Acta, Part A 2019, 206, 632.

[36] Xiao, H. B.; Zhang, Y. Z.; Zhang, W.; Li, S. Z.; Xu, R. H. Sens. Actuators, B 2016, 233, 469.

[37] Jiang, Y.-B.; Gao, C.; Zhang, X.; Yao, J.-S.; Liu, Q.-Z.; Cai, X.-X. J. Mol. Struct. 2018, 1163, 33.

[38] Pal, S.; Roy, D.; Bar, N.; Chowdhury, S.; Chowdhury, P. Polymer 2020, 191, 122292

[39] Wang, X. N.; Zuo, Y. J.; Zhang, Y.; Yang, T. X.; Lin, W. Y. Analyst $2019,144,5373$.

[40] Thanzeel, F. Y.; Sripada, A.; Wolf, C. J. Am. Chem. Soc. 2019, 141, 16382

[41] Pang, C.-M.; Chen, S.-H.; Cao, X.-Y.; Zhang, J.-R.; Xiao, Y.; Li, X.-D.; Luo, S.-H.; Wang, Z.-Y. J. Photochem. Photobiol., A 2020, 403, 112835

[42] Chen, S.-H.; Pang, C.-M.; Chen, X.-Y.; Yan, Z.-H.; Huang, S.-M.; Li, X.-D.; Zhong, Y.-T.; Wang, Z.-Y. Chin. J. Org. Chem. 2019, 39, 1846 (in Chinese).

(陈思鸿，庞楚明，陈孝云，严智浩，黄诗敏，李香弟，钟雅婷， 汪朝阳，有机化学, 2019, 39, 1846.)

[43] Erdemir, S.; Malkondu, S. Sens. Actuators, B 2013, 188, 1225

[44] Li, W.; Tian, X. H.; Huang, B.; Li, H. J.; Zhao, X. Y.; Gao, S.; Zheng, J.; Zhang, X. Z.; Zhou, H. P.; Tian, Y. P.; Wu, J. Biosens. Bioelectron. 2016, 77, 530

[45] Shen, B. X.; Qian, Y. ChemistrySelect 2017, 2, 2406.

[46] Kundu, A.; Anthony, S. P. Spectrochim. Acta, Part A 2018, 189, 342 . 
[47] Wang, L.; Yang, X. D.; Chen, X. L.; Zhou, Y. P.; Lu, X. D.; Yan, C. G.; Xu, Y. K.; Liu, R. Y.; Qu, J. Q. Mater. Sci. Eng., C 2017, 72, 551.

[48] Mathivanan, M.; Tharmalingam, B.; Mani, K. S.; Thiagarajan, V.; Murugesapandian, B. Spectrochim. Acta, Part A 2020, 234, 118235.

[49] Zhang, X.; Sun, P.; Li, F.; Li, H.; Zhou, H. P.; Wang, H.; Zhang, B. W.; Pan, Z. W.; Tian, Y. P.; Zhang, X. J. Sens. Actuators, B 2018, 255,366 .

[50] Shen, B.-X.; Qian, Y. J. Mater. Chem. B 2016, 4, 7549.

[51] Erdemir, S. Sens. Actuators, B 2019, 290, 558.

[52] Sathiyan, G.; Chatterjee, S.; Sen, P.; Garg, A.; Gupta, R. K.; Singh, A. ChemistrySelect 2019, 4, 11718.

[53] Thamaraiselvi, P.; Duraipandy, N.; Kiran, M. S.; Easwaramoorthi, S. ACS Sustainable Chem. Eng. 2019, 7, 9865.

[54] Wu, Y.-C.; You, J.-Y.; Jiang, K.; Xie, J.-C.; Li, S.-L.; Cao, D. R.; Wang, Z.-Y. Dyes Pigm. 2017, 140, 47.

[55] Wu, Y.-C.; Huo, J.-P.; Cao, L.; Ding, S.; Wang, L.-Y.; Cao, D. R.; Wang, Z.-Y. Sens. Actuators, B 2016, 237, 865

[56] Beneto, A. J.; Siva, A. Photochem. Photobiol. Sci. 2017, 16, 255.

[57] Tamilarasan, D.; Suhasini, R.; Thiagarajan, V.; Balamurugan, R. Eur. J. Org. Chem. 2020, 993.

[58] Ozdemir, A.; Erdemir, S. J. Photochem. Photobiol., A 2020, 390, 112328.

[59] Li, Q. Y.; Wang, Z. C.; Song, W. W.; Ma, H. L.; Dong, J. Y.; Quan, Y. Y.; Ye, X. X.; Huang, Z.-S. Dyes Pigm. 2019, 161, 389.

[60] Zheng, Z.-H.; Li, Z.-K.; Song, L.-J.; Wang, Q.-W.; Huang, Q.-F.; Yang, L. Sensors 2017, 17, 405.

[61] Zhang, Q. S.; Zhang, J.; Zuo, H. J.; Wang, C. Y.; Shen, Y. J. Tetrahedron 2016, 72, 1244.

[62] Yang, C. J.; Wang, X. C.; Xu, Z. G.; Wang, M. F. Sens. Actuators, B 2017, 245, 845 .

[63] Liu, Y. J.; Qiu, D. L.; Pan, H.; Li, M. N.; Chen, H. B.; Li, H. M. J. Photochem. Photobiol., A 2018, 364, 151.

[64] Zhang, H.-M.; Wu, Y.-C.; You, J.-Y.; Cao, L.; Ding, S.; Jiang, K.; Wang, Z.-Y. Chin. J. Org. Chem. 2016, 36, 2559 (in Chinese). (张惠敏, 吴彦城, 尤嘉宜, 曹梁, 丁沙, 蒋凯, 汪朝阳, 有机化 学, 2016, 36, 2559.)

[65] Ponnuvel, K.; Padmini, V. J. Lumin. 2016, 169, 289.

[66] Che, W. L.; Li, G. F.; Zhang, J. X.; Geng, Y.; Xie, Z. G.; Zhu, D. X.; Su, Z. M. J. Photochem. Photobiol., A 2018, 358, 274.

[67] Zhang, D. S.; Liu, A. K.; Ji, R. X.; Dong, J.; Ge, Y. Q. Anal. Chim. Acta 2019, 1055, 133

[68] Cheng, X. H.; He, P.; Zhong, Z. C.; Liang, G. J. Luminescence 2016, 31, 1372 .

[69] Liu, K. Y.; Chen, Y. L.; Sun, H.; Wang, S. J.; Kong, F. G. J. Mater. Chem. B 2018, 6, 7060.

[70] Wang, L. Y.; Li, L. Q.; Cao, D. R. Sens. Actuators, B 2017, 241, 1224.

[71] Jiang, Y. H.; Zhang, S.; Wang, B. X.; Qian, T.; Jin, C.; Wu, S. S.; Shen, J. Tetrahedron 2018, 74, 5733.

[72] Xu, C.; Qian, Y.; Qi, Z.-Q.; Lu, C.-G.; Cui, Y.-P. New J. Chem. 2018, $42,6910$.

[73] Xu, X.-X.; Qian, Y. Spectrochim. Acta, Part A 2017, 183, 356.

[74] Xu, X.-X.; Qian, Y. New J. Chem. 2017, 41, 9607.

[75] Wang, T.; Shah, I.; Yang, Z. M.; Yin, W. D.; Zhang, S. X.; Yang, Y.; Yin, P.; Ma, H. C. Anal. Chem. 2020, 92, 2824.

[76] Ma, H. C.; Yang, M. Y.; Zhang, S. X.; Yin, P.; Wang. T.; Yang, Y.; Lei, Z. Q.; Ma, Y. C.; Qin, Y. F.; Yang, Z. M. Analyst 2019, 144, 536.

[77] Cao, L.; Xiong, J.-F.; Wu, Y.-C.; Ding, S.; Li, M.-B.; Xie, F.; Ma, Z.-H.; Wang, Z.-Y. Chin. J. Org. Chem. 2016, 36, 2053 (in Chinese).

(曹梁，熊金锋，吴彦城，丁沙，李铭冰，谢芬，马志晗，汪朝阳， 有机化学, 2016, 36, 2053.)

[78] Wang, B.-W.; Jiang, K.; Li, J.-X.; Luo, S.-H.; Wang, Z.-Y.; Jiang, H.-F. Angew. Chem., Int. Ed. 2020, 59, 2338

[79] Chen, S.-H.; Jiang, K.; Lin, J. Y.; Yang, K.; Cao, X.-Y.; Luo, X.-Y.;
Wang, Z.-Y. J. Mater. Chem. C 2020, $8,8257$.

[80] Ma, H. W.; Li, F.; Li, P.; Wang, H. L.; Zhang, M.; Zhang, G.; Baumgarten, M.; Mullen, K. Adv. Funct. Mater. 2016, 26, 2025.

[81] Ji, N.-N.; Shi, Z.-Q.; Hu, H.-L.; Zheng, H.-G. Dalton Trans. 2018, $47,7222$.

[82] Xiong, J.-F.; Li, J.-X.; Mo, G.-Z.; Huo, J.-P.; Liu, J.-Y.; Chen, X.-Y.; Wang, Z.-Y. J. Org. Chem. 2014, 79, 11619.

[83] Wu, Y.-C.; Luo, S.-H.; Cao, L.; Jiang, K.; Wang, L.-Y.; Xie, J.-C.; Wang, Z.-Y. Anal. Chim. Acta 2017, 976, 74.

[84] Jiang, K.; Luo, S.-H.; Pang, C.-M.; Wang, B.-W.; Wu, H.-Q.; Wang, Z.-Y. Dyes Pigm. 2019, 162, 367.

[85] Sathiyan, G.; Balasubramaniam, B.; Ranjan, S.; Chatterjee, S.; Sen, P.; Garg, A.; Gupta, R. K.; Singh, A. Mater. Today Chem. 2019, 12, 178.

[86] Wu, H. T.; Tao, F. R.; Cui, Y. Z.; Guo, L. Mater. Chem. Phys. 2020, 240,122141

[87] Doan, B. K.; Tran, T. V. T.; Nguyen, T. H.; Luu, T. H.; Nguyen, L. T. T; Van, T. P.; Nguyen, V. M.; Nguyen, H. J. Photochem. Photobiol., A 2020, 394, 112496.

[88] Chang, Z.-F.; Jing, L.-M.; Liu, Y.-Y.; Liu, J.-J.; Ye, Y.-C.; Zhao, Y.-S.; Wang, J.-L. J. Mater. Chem. C 2016, 4, 8407.

[89] Wang, N.; Arulkumar, M.; Chen, X.-Y.; Wang, B.-W.; Chen, S.-H.; Yao, C.; Wang, Z.-Y. Chin. J. Org. Chem. 2019, 39, 2771 (in Chinese).

(王能, Mani Arulkumar，陈孝云，王柏文，陈思鸿，姚辰，汪朝 阳, 有机化学, 2019, 39, 2771.)

[90] Chen, S.-H.; Jiang, K.; Xiao, Y.; Cao, X.-Y.; Arulkumar, M.; Wang, Z.-Y. Dyes Pigm. 2020, 175, 108157.

[91] Verbitskiy, E. V.; Kvashnin, Y. A.; Baranova, A. A.; Khokhlov, K. O.; Chuvashov, R. D.; Schapov, I. E.; Yakovleva, Y. A.; Zhilina, E. F.; Shchepochkin, A. V.; Makarova, N. I.; Vetrova, E. V.; Metelitsa, A. V.; Rusinov, G. L.; Chupakhin, O. N.; Charushin, V. N. Dyes Pigm. 2020, 178, 108344.

[92] Zhang, W. J.; Yin, C. X.; Zhang, Y. B.; Chao, J. B.; Huo, F. J. Sens. Actuators, B 2016, 233, 307.

[93] Bu, L. L.; Chen, J. Q.; Wei, X. D.; Li, X.; Agren, H.; Xie, Y. S. Dyes Pigm. 2017, 136, 724.

[94] Xia, Y.; Zhang, H. H.; Zhu, X. J.; Fang, M.; Yang, M. D.; Zhang, Q.; Li, X. W.; Zhou, H. P.; Yang, X. Y.; Tian, Y. P. Dyes Pigm. 2019, $163,441$.

[95] Sun, J. J.; Cao, J.; Cheng, H. L.; Wang, J. C.; Wang, S. X. Chem. Res. Chin. Univ. 2019, 35, 990

[96] Duan, Y. K.; Ding, G.; Yao, M. Y.; Wang, Q.; Guo, H.; Wang, X. C.; Zhang, Y. F.; Li, J. Y.; Li, X. J.; Qin, X. Z. Spectrochim. Acta, Part A 2020, 236, 118348

[97] Jiang, N.; Wang, B.; Liu, T.; Liu, Q. Y.; Wei, Q.; Xing, Y. J.; Zheng, G. X. Anal. Methods 2019, 11, 232.

[98] Xu, L. F.; Ni, L.; Sun, L. H.; Zeng, F.; Wu, S. Z. Analyst 2019, 144, 6570.

[99] Li, Q. Y.; Wang, Z. C.; Zhao, M.; Hong, Y. P.; Jin, Q. W.; Yao, S. Y.; Zheng, C. L.; Quan, Y.-Y.; Ye, X. X.; Huang, Z.-S. Sens. Actuators, B 2019, 298, 126898.

[100] Ma, H. C.; Qi, C. X.; Cao, H. Y.; Zhang, Z. W.; Yang, Z. M.; Zhang, B.; Chen, C.; Lei, Z. Q. Chem.-Asian J. 2016, 11, 58.

[101] Aich, K.; Das, S.; Gharami, S.; Patra, L.; Mondal, T. K. New J. Chem. 2017, 41, 12562.

[102] Wu, Y.; Jin, P. W.; Gu, K. Z.; Shi, C. X.; Guo, Z. Q.; Yu, Z.-Q.; Zhu, W.-H. Chem. Commun. 2019, 55, 4087.

[103] Wang, X. C.; Ding, G.; Duan, Y. K.; Zhu, Y. J.; Zhu, G. S.; Wang, M.; Li, X. J.; Zhang, Y. F.; Qin, X. Z.; Hung, C.-H. Talanta 2020, 217,121029

[104] Wang, Z.-Y.; Chen, S.-H.; Jiang, K. CN 111039892, 2020

[105] Han, M. K.; Chen, M.; Ebendorff-Heidepriem, H.; Fang, C.; Qin, A. J.; Zhang, H.; Tang, B. Z.; Tang, Y. H.; Ruan, Y. L. RSC $A d v$ 2016, 6, 82186 .

[106] Wu, Z. H.; Fu, X. B.; Wang, Y. Sens. Actuators, B 2017, 245, 406. 